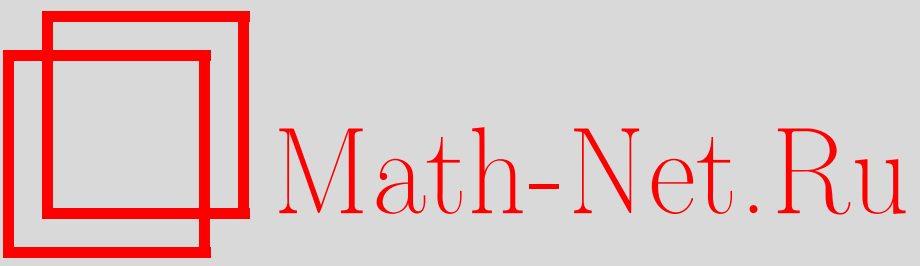

Б. А. Погорелов, М. А. Пудовкина, Свойства графов орбиталов надгрупп группы Джевонса, Матем. вопр. криптогр., 2010, том 1, выпуск 1, 55-83

DOI: https://doi.org/10.4213/mvk4

Использование Общероссийского математического портала Math-Net.Ru подразумевает, что вы прочитали и согласны с пользовательским соглашением

http://www.mathnet.ru/rus/agreement

Параметры загрузки:

IP : 52.87 .193 .239

26 апреля 2023 г., 11:58:45 


\author{
МАТЕМАТИЧЕСКИЕ ВОПРОСЫ КРИПТОГРАФИИ \\ 2010 T. 1 № 1 C. 55-84
}

УДК 512.542 .76

\title{
Свойства графов орбиталов надгрупп группы Джевонса
}

\author{
Б. А. Погорелов ${ }^{1)}$, М. А. Пудовкина ${ }^{2)}$ \\ 1) Академия криптографии Российской Федерации, Москва \\ ${ }^{2)}$ Московский инженерно-физический институт, Москва
}

Получено 22.IV.2010

Рассматриваются графы орбиталов подсхем схемы Хемминга. Среди таких графов описываются дистанционно-транзитивные, дистанционно-регулярные, антиподальные и бипримитивные графы. Описаны также группы автоморфизмов указанных графов орбиталов, их метрики и факторметрики.

Ключевые слова: ассоциативная схема отношений, граф орбитала, метрика, подметрика, дистанцинно-транзитивный граф, дистанционно-регулярный граф.

\section{Properties of graphs of orbitals for overgroups of the Jevons group}

\section{B. A. Pogorelov ${ }^{1)}$, M. A. Pudovkina ${ }^{2)}$ \\ ${ }^{1)}$ Academy of Cryptography of Russian Federation, Moscow \\ ${ }^{2)}$ Moscow Engineering Physics Institute}

Abstract. In this paper we consider properties of graphs of orbitals for sub-schemes of the Hamming scheme. We describe distance-transitive, distance-regular, antipodal and bipartite graphs of orbitals. Automorphism groups of the graphs of orbitals, their metrics, quotient metric and corresponding properties are investigated also.

Keywords: association scheme, orbital graph, metric, submetric, distance-transitive graph, distance-regular graph.

Citation: Mathematical Aspects of Cryptography, 2010, vol. 1, no. 1, pp. 55-84 (Russian). 


\section{1. Введение}

Напомним [3], что целочисленная метрика $\mu: V_{n} \times V_{n} \rightarrow \mathbb{N}$, где $V_{n}=(\mathrm{GF}(2))^{n}$, называется подметрикой метрики Хемминга $\chi$, если для любых векторов $\alpha, \beta, \gamma, \delta \in V_{n}$ она удовлетворяет условиям:

1) если $\chi(\alpha, \beta)=\chi(\gamma, \delta)$, то $\mu(\alpha, \beta)=\mu(\gamma, \delta)$;

2) $\mu(\alpha, \beta) \leq \chi(\alpha, \beta)$.

Группой изометрий метрики Хемминга является группа Джевонса $S_{2} \uparrow S_{n}$. Надгруппы группы Джевонса и подметрики метрики Хемминга часто возникают при исследовании различных объектов в дискретной математике и криптографии.

Любому неориентированному связному графу без кратных рёбер и петель (далее - просто графу) соответствует метрика на множестве вершин, задающаяся как расстояние между вершинами. Назовём метрику групповой, если она построена по графу орбитала группы. В работах $[4,5]$ установлено соответствие между подсхемами схемы Хемминга, описанными в работе [2], групповыми подметриками метрики Хемминга, соответствующими орбиталам надгрупп, а также группами автоморфизмов подсхем и группами $G$ изометрий подметрик орбиталов, содержащих произвольные пары векторов $(\alpha, \beta) \in V_{n} \times V_{n}, S_{2} \uparrow S_{n} \leq G \leq S_{2^{n}}$. Такие орбиталы будем называть орбиталами подсхемы схемы Хемминга. Среди графов, соответствующих орбиталам надгрупп группы Джевонса, возникают такие интересные классы графов как дистанционно-транзитивные, антиподальные и двудольные. Подобным графам посвящён целый ряд работ ([6, 7, 8, 12, 13, 14 и др.).

В данной работе найдены условия наличия или отсутствия дистанционной транзитивности и регулярности, антиподальности, двудольности у графов орбиталов подсхем схемы Хемминга. Указаны группы автоморфизмов, соответствующих графов их метрики, факторметрики и другие свойства. Также доказана замкнутость множества подсхем схемы Хемминга относительно введённых унарных операций «антиподальности», «двудольности».

\section{1. Основные обозначения и понятия}

Обозначим:

- $\mathbb{N}$ - множество всех натуральных чисел;

- $N^{(0)}, N^{(1)}$ - соответственно множество четных и нечетных натуральных чисел;

- $\{\overline{a, b}\}=\{a, a+1, \ldots, b\} \subseteq \mathbb{N}, a<b$; 
- $\{\overline{a, b}\}^{0},\{\overline{a, b}\}^{1}$ - соответственно множество всех чётных и нечётных чисел из множества $\{\overline{a, b}\}$;

- $V_{n}$ - пространство всех $n$-мерных двоичных векторов над полем $G F(2)$;

- $W_{n}$ - подпространство всех векторов чётного веса из $V_{n}$,

- $\bar{W}_{n}=V_{n} \backslash W_{n}$,

- $\|\alpha\|$ - вес (Хемминга) вектора $\alpha \in V_{n}$;

- $H(n, 2)$ - схема отношений Хемминга;

- $\bar{G}$ - образ при естественном гомоморфизме импримитивной группы $G$, действующей на множестве блоков импримитивности;

- $\lceil a\rceil$ — наименьшее целое, большее или равное числу $a$;

- $\lfloor a\rfloor$ - наибольшее целое, меньшее или равное числу $a$;

- $\omega_{b}(\gamma)=\min \{\|\gamma\|, n-\|\gamma\|+b\}, b \in\{-1,0,1\}$;

- $\bar{\mu}(\|\alpha+\beta\|)=\mu(\alpha, \beta)$, где $\alpha, \beta \in V_{n}, \mu-$ подметрика метрики Хемминга и $\bar{\mu}:\{\overline{0, n}\} \rightarrow\{\overline{0, n}\}$;

- $\Delta_{i}$ - множество всех векторов веса $i$ из $V_{n}$,

- $C_{k}=\{i \in\{\overline{1, n-1}\} \mid i \equiv k(\bmod 4)\}, D_{k}=\{i \in\{\overline{1, n}\} \mid i \equiv k(\bmod 4)\}$, $k=0,1,2,3$;

- $C_{0}, C_{1}, C_{2}, C_{3},\{0\},\{n\}$ и $D_{0}, D_{1}, D_{2}, D_{3},\{0\}$ - разбиения множества $\{\overline{0, n}\}$;

- $\Delta_{0}, \Lambda_{0}, \Lambda_{1}, \Lambda_{2}, \Lambda_{3}, \Delta_{n}$ и $\Delta_{0}, \Theta_{0}, \Theta_{1}, \Theta_{2}, \Theta_{3}$ - разбиения пространства $V_{n}$, где $\Theta_{k}=\sum_{i \in D_{k}} \Delta_{i}, \Lambda_{k}=\sum_{i \in C_{k}} \Delta_{i}$ и $k=0,1,2,3$.

Пусть $\Psi$ - произвольный самопарный орбитал подсхемы схемы Хемминга, $\bar{\Psi}=\left(V_{n} ; \Psi\right)-$ граф, соответствующий орбиталу $\Psi, \Psi_{\overrightarrow{0}}=\{\alpha \mid(\overrightarrow{0}, \alpha) \in \Psi\}$. Если $\quad \Psi_{\overrightarrow{0}}=\bigcup_{i \in I} \Delta_{i}, \quad$ где $\quad I=\left\{i_{1}, \ldots, i_{l}\right\} \subseteq\{\overline{1, n}\}, \quad$ то обозначим $\Psi=\Gamma_{i_{1} \cup \ldots \cup i_{l}}$, $\bar{\Gamma}_{i_{1} \cup \ldots \cup i_{l}}=\left(V_{n}, \Gamma_{i_{1} \cup \ldots \cup i_{l}}\right)$. Кроме того, если множество $I$ представимо в виде $I=\bigcup_{j \in J} D_{j} \quad$ или $\quad I=\bigcup_{j \in J} C_{j} \quad$ для $\quad$ некоторого множества индексов 
$J=\left\{j_{1}, \ldots, j_{r}\right\} \subseteq\{0,1,2,3\}, r \leq 4$, то соответственно обозначим $\Psi=\Gamma_{\left[j_{1} \cup \ldots \cup j_{r}\right]}$, $\Psi=\Gamma_{\left(j_{1} \cup \ldots \cup j_{r}\right)}$.

Орбитал $\Psi$ называется нечётным, если $\Delta_{k} \subseteq \Psi_{\overrightarrow{0}}$ для некоторого нечётного числа $k \in\{\overline{1, n}\}$. В противном случае орбитал называется чётным. Если $\Delta_{1} \subseteq \Psi_{\overrightarrow{0}}$, то орбитал $\Psi$ называется базовым и обозначается $\Psi_{1}$.

Будем говорить, что орбитал $\Psi$ принадлежит схеме отношений, если $\Psi$ - «подмножество» схемы отношений. Орбитал $\Psi$ может принадлежать разным подсхемам схемы Хемминга $H(n, 2)$. Каждому нечётному орбиталу $\Psi$ поставим в соответствие такой базовый орбитал $\Psi_{1}$ наибольшей мощности, что орбиталы $\Psi, \Psi_{1}$ принадлежат одной подсхеме схемы Хемминга.

По степени «похожести» (под «похожестью» понимается единообразное описание метрик и их групп изометрий, кроме класса $R_{2}$ ) орбиталы подсхемы схемы Хемминга $H(n, 2)$ можно разбить на следующие семь классов [5]:

$$
\begin{aligned}
& R_{0}=\left\{\Gamma_{1}, \Gamma_{2}, \ldots, \Gamma_{n-1}\right\}, \\
& R_{1}=\left\{\begin{array}{l}
\left\{\Gamma_{(k)}, \Gamma_{[k]} \mid k \in\{0,1,2,3\}\right\}, \text { если } n \in N^{(1)}, \\
\left\{\Gamma_{[k]} \mid k \in\{0,1,2,3\}\right\}, \text { если } n \in N^{(0)},
\end{array}\right. \\
& R_{2}=\left\{\begin{array}{l}
\left\{\Gamma_{(0 \cup 3)}, \Gamma_{(0 \cup 1)}, \Gamma_{(2 \cup 3)}, \Gamma_{(1 \cup 2)}, \Gamma_{[0 \cup 2]}, \Gamma_{(1 \cup 3)}, \Gamma_{[1 \cup 3]}\right\}, \text { если } n \in N^{(1)}, \\
\left\{\Gamma_{[0 \cup 3]}, \Gamma_{[0 \cup 1]}, \Gamma_{[2 \cup 3]}, \Gamma_{[1 \cup 2]}, \Gamma_{(0 \cup 2)}, \Gamma_{[0 \cup 2]}, \Gamma_{[1 \cup 3]}\right\}, \text { если } n \in N^{(0)},
\end{array}\right. \\
& R_{3}=\left\{\begin{array}{l}
\left.\left\{\Gamma_{k \cup n+b-k} \mid b \in\{-1,0,1\}, k \in\{\overline{1,\lfloor n / 2}]\right\}\right\}, \text { если } n \in N^{(1)}, \\
\left\{\Gamma_{k \cup n+b-k} \mid b \in\{0,1\}, k \in\{\overline{1,\lfloor n / 2]}\}\right\}, \text { если } n \in N^{(0)},
\end{array}\right. \\
& R_{4}=\left\{\Gamma_{2 k-1 \cup 2 k} \mid k \in\{\overline{1,\lfloor n / 2\rfloor}\}\right\} \text {, } \\
& R_{5}=\left\{\begin{array}{l}
\left\{\Gamma_{2 k-1 \cup 2 k \cup n-2 k \cup n-2 k+1} \mid k \in\{\overline{1,\lfloor n / 4]}\}\right\}, \text { если } n \in N^{(1)}, \\
\left\{\Gamma_{2 k-1 \cup 2 k \cup n-2 k+1 \cup n-2 k+2} \mid k \in\{\overline{1,\lfloor n / 4]}\}\right\}, \text { если } n \in N^{(0)},
\end{array}\right. \\
& R_{6}=\left\{\Gamma_{(0 \cup 1 \cup 2 \cup 3)}, \Gamma_{[0 \cup 1 \cup 2 \cup 3]}\right\} \text {. }
\end{aligned}
$$


Имеют место равенства:

$$
\begin{aligned}
& \Gamma_{(k)}=\Gamma_{[k]}, \text { если } k \not \equiv n(\bmod 2) ; \\
& \Gamma_{[1 \cup 3]}=\Gamma_{(1 \cup 3)}, \text { если } n \in N^{(0)} ; \\
& \Gamma_{[0 \cup 2]}=\Gamma_{(0 \cup 2)}, \text { если } n \in N^{(1)}
\end{aligned}
$$

Метрику, соответствующую $i$-му классу, будем записывать в виде $\rho_{*}^{(i)}$, $i=\overline{0,6}$. При этом положим:

$$
\begin{aligned}
& \chi_{k}=\rho_{k}^{(0)}-\text { метрика орбитала } \Gamma_{k} \in R_{0}, k \in\{\overline{1, n-1}\} ; \\
& \rho_{(k)}^{(1)}-\text { метрика орбитала } \Gamma_{(k)} \in R_{1}, k \in\{0,1,2,3\} ; \\
& \rho_{[k]}^{(1)}-\text { метрика орбитала } \Gamma_{[k]} \in R_{1}, k \in\{0,1,2,3\} ; \\
& \rho_{i, b, 1}^{(2)}-\text { метрика орбитала } \Gamma_{(i \cup i+b)} \in R_{2}, i \in\{0,1,2\}, b \in\{1,2,3\}, \\
& \quad \text { и } 0 \leq i<i+b<4 ; \\
& \rho_{i, b, 2}^{(2)}-\text { метрика орбитала } \Gamma_{[i \cup i+b]} \in R_{2}, i \in\{0,1,2\}, b \in\{1,2,3\}, \\
& \quad \text { и } 0 \leq i<i+b<4 ; \\
& \rho_{k, b}^{(3)}-\text { метрика орбитала } \Gamma_{k \cup n+b-k} \in R_{3}, b \in\{-1,0,1\}, k=1, \ldots,\lfloor n / 2\rfloor ; \\
& \rho_{k}^{(4)}-\text { метрика орбитала } \Gamma_{2 k-1 \cup 2 k} \in R_{4}, k=1, \ldots,\lfloor n / 2\rfloor ; \\
& \rho_{k, 1}^{(5)}-\text { метрики орбитала } \Gamma_{2 k-1 \cup 2 k \cup n-2 k \cup n-2 k+1} \in R_{5} \text { при } n \in N^{(1)}, \\
& \quad k=1, \ldots,\lfloor n / 4\rfloor ; \\
& \rho_{k, 2}^{(5)}-\text { метрики орбитала } \Gamma_{2 k-1 \cup 2 k \cup n-2 k+1 \cup n-2 k+2} \in R_{5} \text { при } n \in N^{(0)}, \\
& \quad k=1, \ldots,\lfloor n / 4\rfloor ; \\
& \rho_{1}^{(6)}-\text { метрики орбитала } \Gamma_{(0 \cup 1 \cup 2 \cup 3)} \in R_{6} ; \\
& \rho_{2}^{(6)}-\text { метрики орбитала } \Gamma_{[0 \cup 1 \cup 2 \cup 3]} \in R_{6} ; \\
& \rho_{1}^{(6)}(\alpha, \beta)=\left\{\begin{array}{l}
1, \text { если }\|\alpha+\beta\| \in\{1, \ldots, n-1\}, \\
2, \text { если }\|\alpha+\beta\|=n, \\
\rho_{2}^{(6)}(\alpha, \beta)=1 \text { для любых векторов } \alpha, \beta \in V_{n} .
\end{array}\right.
\end{aligned}
$$

Обозначим через $\chi_{k}(\alpha, \beta)$ расстояние между вершинами $\alpha, \beta$ в графе $\bar{\Gamma}_{k}, \chi(\alpha, \beta)=\chi_{1}(\alpha, \beta)$. 


\section{2. Дистанционно-транзитивные графы нечетных орбиталов}

Напомним, что граф $\Gamma=(X, Y)$ называется дистанционно-транзитивным, если для любых вершин $u, u^{\prime}, v, v^{\prime} \in X$ таких, что $d\left(u, u^{\prime}\right)=d\left(v, v^{\prime}\right)$, существует элемент $g \in \operatorname{Aut}(\Gamma)$, удовлетворяющий равенствам $v=u^{g}, v^{\prime}=u^{\prime g}$. Граф $\Gamma=(X, Y)$ называется вершинно-транзитивным, если группа $\operatorname{Aut}(\Gamma)$ действует на множестве $X$ транзитивно.

Различным аспектам классификации дистанционно-транзитивных графов посвящены работы $[12,8,9,17,16,14]$. Очевидно, что связный граф $\Gamma=(X, Y)$ диаметра $b \geq 1$ является дистанционно-транзитивным тогда и только тогда, когда $\Gamma-$ вершинно-транзитивный и стабилизатор $\operatorname{Aut}(\Gamma)_{v}$ транзитивен на множестве $\Gamma^{i}(v)$ для каждых $v \in X, i \in\{\overline{1, b}\}$, где $\Gamma^{i}(v)=\{\alpha \in X \mid \rho(\alpha, v)=i\}, \rho(\alpha, v)-$ расстояние между вершинами $v, \alpha \in X$ в графе $\Gamma$.

Отсюда непосредственно вытекает следующий критерий дистанционной транзитивности графов, подгруппой группы автоморфизмов которых является $S_{2} \uparrow S_{n}$.

Лемма 2.1. Пусть $Г-$ связный граф с множеством вершин $V_{n} \partial и а$ метра $b \geq 1$ такой, что $S_{2} \uparrow S_{n} \leq \operatorname{Aut}(\Gamma)-$ группа ранга $r$. Пусть также $X_{i}-i$-я орбита стабилизатора $\operatorname{Aut}(\Gamma)_{\overrightarrow{0}}, i=\overline{0, r-1}$. Граф $\Gamma$ является дистанционно-транзитивным тогда и только тогда, когда для каждого $j \in\{\overline{1, b}\}$ выполняется включение $\Gamma^{j}(\overrightarrow{0}) \subseteq X_{i_{j}}$ для некоторого $i_{j} \in\{\overline{0, r-1}\}$.

В таблицах 1 и 3 работы [5] приведены все нечётные орбиталы подсхем схемы Хемминга (см. также приложение).

Опираясь на полученные в [5] метрики орбиталов, описание их групп изометрий и классификацию подорбит групп автоморфизмов подсхем схемы Хемминга, опишем дистанционно-транзитивные графы орбиталов подсхем схемы Хемминга. Отметим, что графы всех чётных орбиталов несвязные.

Теорема 2.2. Дистанционно-транзитивные графы нечётных орбиталов подсхем схемы Хемминга при $n \geq 6$ исчерпываются следующим списком:

a) $\bar{\Gamma}_{1}, \bar{\Gamma}_{1 \cup 2}, \bar{\Gamma}_{1 \cup n}, \bar{\Gamma}_{[1]}, \bar{\Gamma}_{[3]}, \bar{\Gamma}_{[1 \cup 3]}, \bar{\Gamma}_{[0 \cup 1 \cup 2 \cup 3]}, \bar{\Gamma}_{(0 \cup 1 \cup 2 \cup 3)}$;

б) $\bar{\Gamma}_{(0 \cup 1)}, \bar{\Gamma}_{(2 \cup 3)}, \bar{\Gamma}_{(1 \cup 2)}, \bar{\Gamma}_{(0 \cup 3)}, \bar{\Gamma}_{(1 \cup 3)}$ при нечётном $n$;

в) $\bar{\Gamma}_{[0 \cup 1]}, \bar{\Gamma}_{[2 \cup 3]}, \bar{\Gamma}_{[1 \cup 2]}, \bar{\Gamma}_{[0 \cup 3]}, \bar{\Gamma}_{1 \cup 2 \cup n \cup n-1}$ при чётном $n$. 
Среди всех нечётных графов дистанционно-транзитивными являются графы, соответствующие обведённым пунктиром вершинам. Штрихованная линия вложения соответствует случаю $n \equiv 1(\bmod 4)$ или $n \equiv 0(\bmod 4)$, штрихпунктирная - случаю $n \equiv 3(\bmod 4)$ или $n \equiv 2(\bmod 4)$. Любой жирный тип линии соответствует вложению дистанционно-транзитивных графов.

Доказательство состоит из следующих этапов. Для каждого орбитала $\Psi$, приведенного в таблицах 1, 3 [5], выписываются подорбиты $X_{0}, \ldots, X_{r-1}$ группы $\operatorname{Aut}(\bar{\Psi})$ ранга $r$. Затем определяются множества $\bar{\Psi}^{i}(\overrightarrow{0})=\left\{\alpha \in V_{n} \mid \rho(\alpha, \overrightarrow{0})=i\right\}$, где $\rho(\alpha, v)$ - кратчайшее расстояние между вершинами $v, \alpha \in V_{n}$ в графе $\bar{\Psi}$. После чего для каждого $j=1,2, \ldots$ проверяется справедливость включения $\bar{\Psi}^{j}(\overrightarrow{0}) \subseteq X_{i_{j}}$ для некоторого $i_{j} \in\{\overline{0, r-1}\}$.

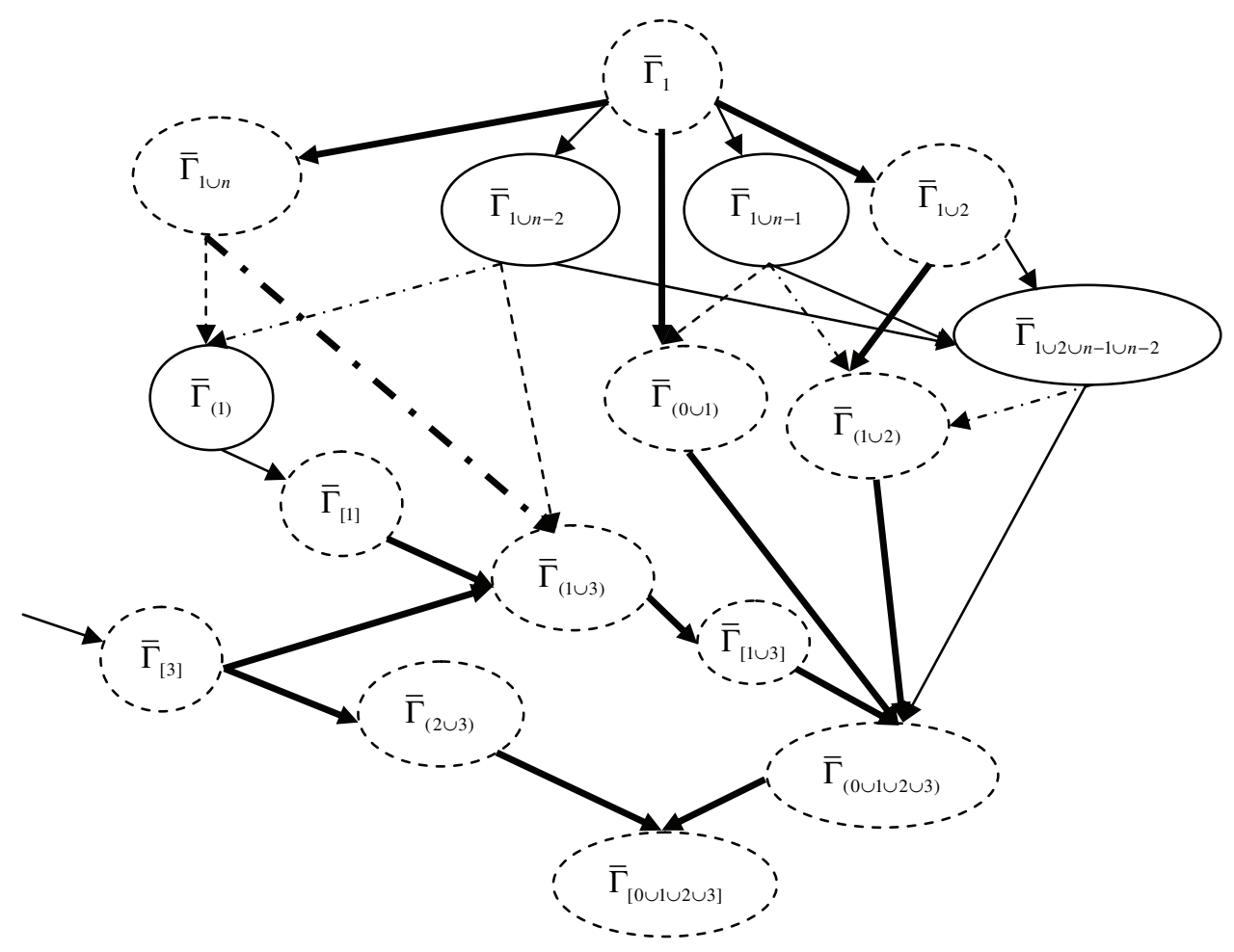

Рис. 1. Граф вложений дистанционно-транзитивных графов нечётных степеней $n$ 


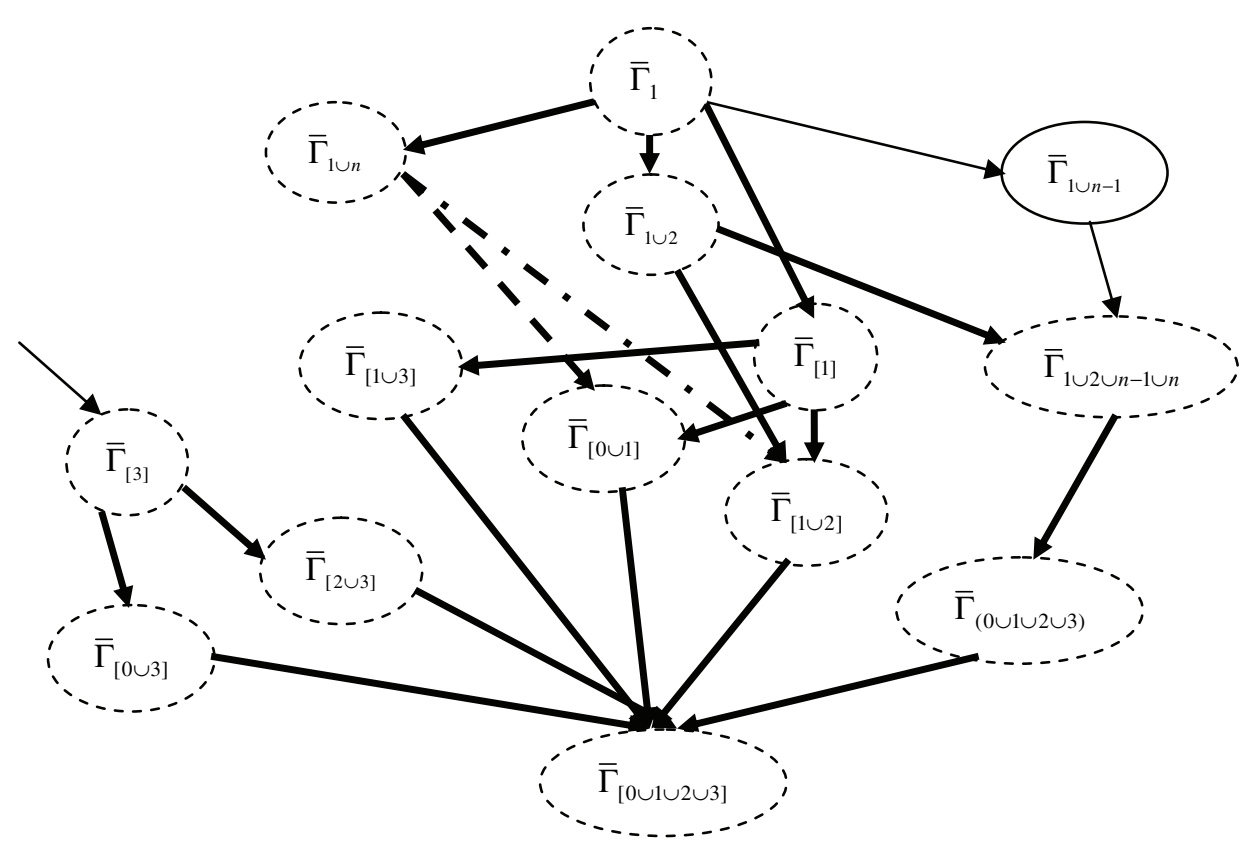

Рис. 2. Граф вложений дистанционно-транзитивных графов чётных степеней $n$

1. Граф $\bar{\Gamma}_{k}$. Известно [1], что граф $\bar{\Gamma}_{1}$ дистанционно-транзитивный. При $k \geq 3$ из работы [5] следует, что $\Delta_{2} \cup \Delta_{2 k} \subseteq \bar{\Gamma}_{k}^{2}(\overrightarrow{0})$ при $k \leq\lfloor n / 2\rfloor$, $\Delta_{2} \cup \Delta_{2(n-k)} \subseteq \bar{\Gamma}_{k}^{2}(\overrightarrow{0})$ при $k>\lfloor n / 2\rfloor$, а орбиты $\operatorname{Aut}\left(\bar{\Gamma}_{k}\right)_{\overrightarrow{0}}$ равны $\Delta_{0}, \Delta_{1}, \Delta_{2}, \ldots$, $\Delta_{2 i-1}, \Delta_{2 i}, \ldots, \Delta_{n}$. Таким образом, граф $\bar{\Gamma}_{k}$ не является дистанционнотранзитивным при нечётном $k \geq 3$.

2. Графы $\bar{\Gamma}_{(1)}, \bar{\Gamma}_{(3)}$. В работе [4] показано, что при $k \in\{1,3\}$ справедливо равенство

$$
\rho_{(k)}^{(1)}(\alpha, \beta)=\left\{\begin{array}{l}
1, \text { если }\|\alpha+\beta\| \equiv k(\bmod 4), \alpha+\beta \neq \overrightarrow{1}, \\
2, \text { если }\|\alpha+\beta\| \equiv 0,2(\bmod 4), \\
3, \text { если }\|\alpha+\beta\| \equiv 3 k(\bmod 4) \text { или } \alpha+\beta=\overrightarrow{1}, n \equiv 1(\bmod 2), \\
4, \text { если } \alpha+\beta=\overrightarrow{1}, n \equiv 0(\bmod 4) .
\end{array}\right.
$$


Из работы [5] следует, что $\operatorname{Aut}\left(\bar{\Gamma}_{(1)}\right)_{\overrightarrow{0}}=\operatorname{Aut}\left(\bar{\Gamma}_{(3)}\right)_{\overrightarrow{0}}$ и группа $\operatorname{Aut}\left(\bar{\Gamma}_{(1)}\right)_{\overrightarrow{0}}$ имеет орбиты $\Delta_{0}, \Lambda_{0}, \Lambda_{1}, \Lambda_{2}, \Lambda_{3}, \Delta_{n}$ при нечётном $n$ и $\Delta_{0}, \Lambda_{1}, \Lambda_{3}, \Lambda_{0} \cup \Lambda_{2}, \Delta_{n}$ при чётном $n$. Для любого $n$ справедливо равенство $\bar{\Gamma}_{(k)}^{2}(\overrightarrow{0})=\Lambda_{2} \cup \Lambda_{0}$. Следовательно, граф $\bar{\Gamma}_{(k)}$ не является дистанционно-транзитивным при нечётном $n$.

Пусть $n$ чётно, тогда

$$
\begin{gathered}
\bar{\Gamma}_{(k)}^{3}(\overrightarrow{0})=\Lambda_{3 k(\bmod 4)}, \\
\bar{\Gamma}_{[k]}^{4}(\overrightarrow{0}) \subseteq \Theta_{0} \text { при } n \equiv 0(\bmod 4), \\
\bar{\Gamma}_{(k)}=\bar{\Gamma}_{[k]} .
\end{gathered}
$$

Значит, $\bar{\Gamma}_{(k)}$ - дистанционно-транзитивный при чётном $n$.

3. Графы $\bar{\Gamma}_{[1]}, \bar{\Gamma}_{[3]}$. В работе [4] показано, что при $k \in\{1,3\}$ справедливо равенство

$$
\rho_{[k]}^{(1)}(\alpha, \beta)=\left\{\begin{array}{l}
1, \text { если }\|\alpha+\beta\| \equiv k(\bmod 4), \\
2, \text { если }\|\alpha+\beta\| \equiv 0,2(\bmod 4), \\
3, \text { если }\|\alpha+\beta\| \equiv k+2(\bmod 4), \\
4, \text { если } \alpha+\beta=\overrightarrow{1}, n \equiv 0(\bmod 4) .
\end{array}\right.
$$

Случай чётного $n$ рассмотрен в пункте 2 . При нечётном $n$ из работы [5] следует, что $\operatorname{Aut}\left(\bar{\Gamma}_{[1]}\right)_{\overline{0}}=\operatorname{Aut}\left(\bar{\Gamma}_{[3]}\right)_{\overline{0}}$ и группа $\operatorname{Aut}\left(\bar{\Gamma}_{[1]}\right)_{\overline{0}}$ имеет орбиты $\Delta_{0}, \Lambda_{0} \cup \Lambda_{2}, \Theta_{1}, \Theta_{3}$. Из соотношений

$$
\begin{gathered}
\bar{\Gamma}_{[k]}^{i}(\overrightarrow{0})=\Theta_{k \cdot i(\bmod 4)}, i=1,3, \\
\bar{\Gamma}_{[k]}^{2}(\overrightarrow{0})=\Lambda_{0} \cup \Lambda_{2}
\end{gathered}
$$

следует дистанционная транзитивность графов $\bar{\Gamma}_{[1]}, \bar{\Gamma}_{[3]}$.

4. Графы $\bar{\Gamma}_{(1 \cup 3)}, \bar{\Gamma}_{[1 \cup 3]}$. В работе [4] показано, что

$$
\rho_{1,2,1}^{(2)}(\alpha, \beta)=\left\{\begin{array}{l}
1, \text { если }\|\beta+\alpha\| \equiv 1(\bmod 2), \alpha+\beta \neq \overrightarrow{1}, \\
2, \text { если }\|\beta+\alpha\| \equiv 0(\bmod 2), \\
3, \text { если } \alpha+\beta=\overrightarrow{1}, n \equiv 1(\bmod 2),
\end{array}\right.
$$




$$
\rho_{1,2,2}^{(2)}(\alpha, \beta)=\left\{\begin{array}{l}
1, \text { если }\|\beta+\alpha\| \equiv 1(\bmod 2), \\
2, \text { если }\|\beta+\alpha\| \equiv 0(\bmod 2) .
\end{array}\right.
$$

Из работы [5] следует, что при нечётном $n$ группа $\operatorname{Aut}\left(\bar{\Gamma}_{(1 \cup 3)}\right)_{\overrightarrow{0}}$ имеет орбиты $\Delta_{0}, \Lambda_{0} \cup \Lambda_{2}, \Lambda_{1} \cup \Lambda_{3}, \Delta_{n}$. Орбиты группы $\operatorname{Aut}\left(\bar{\Gamma}_{[1 \cup 3]}\right)_{\overrightarrow{0}}$ для любого $n$ равны $\Delta_{0}, \Theta_{0} \cup \Theta_{2}, \Lambda_{1} \cup \Lambda_{3}$. Из равенства $\bar{\Gamma}_{[1 \cup 3]}=\bar{\Gamma}_{(1 \cup 3)}$ при чётном $n$ следует, что $\Delta_{0}, \Theta_{0} \cup \Theta_{2}, \Lambda_{1} \cup \Lambda_{3}-$ орбиты группы $\operatorname{Aut}\left(\bar{\Gamma}_{(1 \cup 3)}\right)_{0}$ при чётном $n$. Отсюда получаем, что

$$
\begin{gathered}
\bar{\Gamma}_{[1 \cup 3]}^{2}(\overrightarrow{0})=\Theta_{0} \cup \Theta_{2} \text { при любом } n, \\
\bar{\Gamma}_{(\mathrm{l} \cup 3)}^{2}(\overrightarrow{0})=\Lambda_{0} \cup \Lambda_{2}, \bar{\Gamma}_{(\mathrm{l} \cup 3)}^{3}(\overrightarrow{0})=\Delta_{n} \text { при нечётном } n .
\end{gathered}
$$

Следовательно, графы $\bar{\Gamma}_{(1 \cup 3)}, \bar{\Gamma}_{\text {[1ч3] }}$ - дистанционно-транзитивные.

5. Графы $\bar{\Gamma}_{(0 \cup 1)}, \bar{\Gamma}_{(2 \cup 3)}$, нечётное $n$. В работе [4] показано, что при $i \in\{0,2\}$ справедливо равенство:

$$
\rho_{i, 1,1}^{(2)}(\alpha, \beta)=\left\{\begin{array}{l}
1, \text { если }\|\beta+\alpha\| \equiv i, i+1(\bmod 4), \alpha+\beta \neq \overrightarrow{1}, \\
2, \text { если }\|\beta+\alpha\| \equiv i+2, i+3(\bmod 4) \text { или } \alpha+\beta=\overrightarrow{1}, n \equiv 3(\bmod 4), \\
3, \text { если } \alpha+\beta=\overrightarrow{1}, n \equiv 3(\bmod 4) .
\end{array}\right.
$$

Из работы [5] следует, что $\operatorname{Aut}\left(\bar{\Gamma}_{(0 \cup 1)}\right)_{\overline{0}}=\operatorname{Aut}\left(\bar{\Gamma}_{(2 \cup 3)}\right)_{\overrightarrow{0}} \quad$ и $\quad$ группа $\operatorname{Aut}\left(\bar{\Gamma}_{(0 \cup 1)}\right)_{\overline{0}}$ имеет орбиты $\Delta_{0}, \Lambda_{0} \cup \Lambda_{1}, \Lambda_{2} \cup \Lambda_{3}, \Delta_{n}$. Так как

$$
\begin{aligned}
& \bar{\Gamma}_{(i \cup i+1)}^{2}(\overrightarrow{0})=\Lambda_{i+2(\bmod 4)} \cup \Lambda_{i+3(\bmod 4)}, \\
& \bar{\Gamma}_{(i \cup i+1)}^{3}(\overrightarrow{0})=\Delta_{n} \text { при } n \equiv 3(\bmod 4),
\end{aligned}
$$

то графы $\bar{\Gamma}_{(0 \cup 1)}, \bar{\Gamma}_{(2 \cup 3)}$ - дистанционно-транзитивные.

6. Графы $\bar{\Gamma}_{(1 \cup 2)}, \bar{\Gamma}_{(0 \cup 3)}$, нечётное $n$. В работе [4] показано, что при $i \in\{1,3\}$ справедливо равенство:

$$
\rho_{i, 1,1}^{(2)}(\alpha, \beta)=\left\{\begin{array}{l}
1, \text { если }\|\alpha+\beta\| \equiv i, i+1(\bmod 4), \alpha+\beta \neq \overrightarrow{1}, \\
2, \text { если }\|\alpha+\beta\| \equiv i+2, i+3(\bmod 4) \text { или } \alpha+\beta=\overrightarrow{1}, n \equiv 1(\bmod 4), \\
3, \text { если } \alpha+\beta=\overrightarrow{1}, n \equiv 1(\bmod 4) .
\end{array}\right.
$$


Из работы [5] следует, что $\operatorname{Aut}\left(\bar{\Gamma}_{(1 \cup 2)}\right)_{\overline{0}}=\operatorname{Aut}\left(\bar{\Gamma}_{(0 \cup 3)}\right)_{\overrightarrow{0}} \quad$ и $\quad$ группа $\operatorname{Aut}\left(\bar{\Gamma}_{(1 \cup 2)}\right)_{\overrightarrow{0}}$ имеет орбиты $\Delta_{0}, \Lambda_{0} \cup \Lambda_{3}, \Lambda_{1} \cup \Lambda_{2}, \Delta_{n}$. Аналогично п. 5 получаем, что графы $\bar{\Gamma}_{(1 \cup 2)}, \bar{\Gamma}_{(0 \cup 3)}-$ дистанционно-транзитивные.

7. Графы $\bar{\Gamma}_{[0 \cup 1]}, \bar{\Gamma}_{[2 \cup 3]}$, чётное $n$. В работе [4] показано, что при $i \in\{0,2\}$ справедливо равенство:

$$
\rho_{i, 1,2}^{(2)}(\alpha, \beta)=\left\{\begin{array}{l}
1, \text { если }\|\beta+\alpha\| \equiv i, i+1(\bmod 4), \\
2, \text { если }\|\beta+\alpha\| \equiv i+2, i+3(\bmod 4) .
\end{array}\right.
$$

Из работы [5] следует, что группа $\operatorname{Aut}\left(\bar{\Gamma}_{[0 \cup 1]}\right)_{\overline{0}}$ имеет орбиты $\Delta_{0}, \Theta_{0} \cup \Theta_{1}, \Theta_{2} \cup \Theta_{3}$. Значит, графы $\bar{\Gamma}_{[0 \cup 1]}, \bar{\Gamma}_{[2 \cup 3]}$ - дистанционно-транзитивные.

8. Графы $\bar{\Gamma}_{[1 \cup 2]}, \bar{\Gamma}_{[0 \cup 3]}$, чётное $n$. В работе [4] показано, что при $i \in\{1,3\}$ справедливо равенство:

$$
\rho_{i, 1,2}^{(2)}(\alpha, \beta)=\left\{\begin{array}{l}
1, \text { если }\|\alpha+\beta\| \equiv i, i+1(\bmod 4), \\
2, \text { если }\|\alpha+\beta\| \equiv i+2, i+3(\bmod 4) .
\end{array}\right.
$$

Из работы [5] следует, что $\operatorname{Aut}\left(\bar{\Gamma}_{[1 \cup 2]}\right)_{\overrightarrow{0}}=\operatorname{Aut}\left(\bar{\Gamma}_{[0 \cup 3]}\right)_{\overrightarrow{0}} \quad$ и $\quad$ группа $\operatorname{Aut}\left(\bar{\Gamma}_{[0 \cup 3]}\right)_{0}$ имеет орбиты $\Delta_{0}, \Theta_{0} \cup \Theta_{3}, \Theta_{1} \cup \Theta_{2}$. Значит, графы $\bar{\Gamma}_{[1 \cup 2]}, \bar{\Gamma}_{[0 \cup 3]}-$ дистанционно-транзитивные.

9. Граф $\bar{\Gamma}_{k \cup n-k}$. Если $k=1$, то из работы [4] следует, что

$$
\rho_{1,0}^{3}(\alpha, \beta)= \begin{cases}\min \{\|\alpha+\beta\|, & n-\|\alpha+\beta\|\}, \alpha+\beta \neq \overrightarrow{1}, \\ 2, & \alpha+\beta=\overrightarrow{1},\end{cases}
$$

и $\Delta_{0}, \Delta_{1} \cup \Delta_{n-1}, \ldots, \Delta_{k} \cup \Delta_{n-k}, \ldots, \Delta_{n}-$ орбиты группы $\operatorname{Aut}\left(\bar{\Gamma}_{1 \cup n-1}\right)_{\overline{0}}$. Значит, $\bar{\Gamma}_{1 \cup n-1}^{2}(\overrightarrow{0})=\left(\Delta_{2} \cup \Delta_{n-2}\right) \cup \Delta_{n} \quad$ и $\quad$ граф $\quad \bar{\Gamma}_{1 \cup n-1} \quad$ не $\quad$ является дистанционнотранзитивным.

Пусть $k>1$. Тогда из работы [5] следует, что $\Delta_{2} \cup \Delta_{2 k} \subseteq \bar{\Gamma}_{k \cup n-k}^{2}(\overrightarrow{0})$ при $k \leq\lfloor n / 2\rfloor, \quad \Delta_{2} \cup \Delta_{2(n-k)} \subseteq \bar{\Gamma}_{k \cup n-k}^{2}(\overrightarrow{0}) \quad$ при $k>\lfloor n / 2\rfloor, \quad$ а орбиты группы $\operatorname{Aut}\left(\bar{\Gamma}_{k \cup n-k}\right)_{\overline{0}}$ равны $\Delta_{0}, \Delta_{1} \cup \Delta_{n-1}, \ldots, \Delta_{i} \cup \Delta_{n-i}, \ldots$. Таким образом, граф $\bar{\Gamma}_{k \cup n-k}$ не является дистанционно-транзитивным. 
10. Граф $\bar{\Gamma}_{k \cup n-k+1}$. Пусть $k=1$. Из работы [5] следует, что

$$
\rho_{1,1}^{3}(\alpha, \beta)=\min \{\|\alpha+\beta\|, n+1-\|\alpha+\beta\|\},
$$

a орбиты группы $\operatorname{Aut}\left(\bar{\Gamma}_{1 \cup n}\right)_{\overline{0}}$ есть $\Delta_{0}, \Delta_{1} \cup \Delta_{n}, \ldots, \Delta_{k} \cup \Delta_{n+1-k}, \ldots$. Значит, $\bar{\Gamma}_{1 \cup n}^{k}(\overrightarrow{0})=\Delta_{k} \cup \Delta_{n+1-k}$ и граф $\bar{\Gamma}_{1 \cup n}-$ дистанционно-транзитивный.

Если $n, k$ нечётны, $n+1=2 k$, то из пункта 1 получаем, что граф $\bar{\Gamma}_{k \cup n-k+1}$ не дистанционно-транзитивен.

В остальных случаях из работы [5] следует, что $\Delta_{2} \cup \Delta_{2 k} \subseteq \bar{\Gamma}_{k \cup n-k+1}^{2}(\overrightarrow{0})$ при $k \leq\lfloor n / 2\rfloor, \Delta_{2} \cup \Delta_{2(n-k)} \subseteq \bar{\Gamma}_{k \cup n-k+1}^{2}(\overrightarrow{0})$ при $k>\lfloor n / 2\rfloor$, а орбиты группы $\operatorname{Aut}\left(\bar{\Gamma}_{k \cup n-k+1}\right)_{\overline{0}}$ равны $\Delta_{0}, \Delta_{1} \cup \Delta_{n}, \ldots, \Delta_{k} \cup \Delta_{n+1-k}, \ldots$. Значит, граф $\bar{\Gamma}_{k \cup n-k+1}$ не является дистанционно-транзитивным при $k>1$.

11. Граф $\bar{\Gamma}_{k \cup n-k-1}$ при нечётном $k$. Пусть $k=1$. Из работы [5] следует, что $\rho_{1,-1}^{3}(\alpha, \beta)=2 \quad$ при $\quad\|\alpha+\beta\| \in\{2,4, n-1, n-3\} \quad$ и орбиты группы $\operatorname{Aut}\left(\bar{\Gamma}_{1 \cup n-2}\right)_{\hat{0}}$ равны $\Delta_{0}, \ldots, \Delta_{2 k} \cup \Delta_{n+1-2 k}, \ldots, \Delta_{2 k-1} \cup \Delta_{n-2 k}, \ldots, \Delta_{n}$. Так как

$$
\bar{\Gamma}_{1 \cup n-2}^{2}(\overrightarrow{0})=\left(\Delta_{2} \cup \Delta_{n-1}\right) \cup\left(\Delta_{4} \cup \Delta_{n-3}\right),
$$

то граф $\bar{\Gamma}_{1 \cup n-2}$ не является дистанционно-транзитивным.

Если $n, k$ нечётны, $n-1=2 k$, то из пункта 1 получаем, что граф $\bar{\Gamma}_{k \cup n-k-1}$ не дистанционно-транзитивен. В остальных случаях из работы [5] следует, что $\Delta_{2} \cup \Delta_{2 k} \subseteq \bar{\Gamma}_{k \cup n-k-1}^{2}(\overrightarrow{0})$ при $k \leq\lfloor n / 2\rfloor, \Delta_{2} \cup \Delta_{2(n-k)} \subseteq \bar{\Gamma}_{k \cup n-k-1}^{2}(\overrightarrow{0})$ при $k>\lfloor n / 2\rfloor$ и орбиты $\operatorname{Aut}\left(\bar{\Gamma}_{k \cup n-k-1}\right)_{\overline{0}}$ равны

$$
\Delta_{0}, \Delta_{2} \cup \Delta_{n}, \ldots, \Delta_{2 i} \cup \Delta_{n+1-2 i}, \ldots, \Delta_{1} \cup \Delta_{n-2}, \ldots, \Delta_{2 i-1} \cup \Delta_{n-2 i}, \ldots, \Delta_{n} .
$$

Таким образом, граф $\bar{\Gamma}_{k \cup n-k+1}$ не является дистанционно-транзитивным.

12. Граф $\bar{\Gamma}_{2 k-1 \cup 2 k}$. Пусть $k=1$. Из работы [5] следует, что $\rho_{k}^{(4)}(\alpha, \beta)=\lceil\|\alpha+\beta\| / 2\rceil, \quad \Delta_{0}, \Delta_{1} \cup \Delta_{2}, \ldots, \Delta_{2 k-1} \cup \Delta_{2 k}, \ldots$ - орбиты группы $\operatorname{Aut}\left(\bar{\Gamma}_{1 \cup 2}\right)_{\overline{0}}$. Значит, $\bar{\Gamma}_{1 \cup 2}^{i}(\overrightarrow{0})=\Delta_{2 i-1} \cup \Delta_{2 i}, i=1,2, \ldots$, и граф $\bar{\Gamma}_{1 \cup 2}-$ дистанционно-транзитивный.

При $k \geq 2$ из работы [5] следует, что

$$
\begin{gathered}
\Delta_{2} \cup \Delta_{4 k} \subseteq \bar{\Gamma}_{2 k-1 \cup 2 k}^{2}(\overrightarrow{0}) \text { при } k \leq\lfloor n / 4\rfloor, \\
\Delta_{2} \cup \Delta_{2 n-4 k} \subseteq \bar{\Gamma}_{2 k-1 \cup 2 k}^{2}(\overrightarrow{0}) \text { при } k>\lfloor n / 4\rfloor .
\end{gathered}
$$


Орбиты группы $\operatorname{Aut}\left(\bar{\Gamma}_{2 k-1 \cup 2 k}\right)_{0}$ равны $\Delta_{0}, \Delta_{1} \cup \Delta_{2}, \ldots, \Delta_{2 i-1} \cup \Delta_{2 i}, \ldots$. Значит, граф $\bar{\Gamma}_{2 k-1 \cup 2 k}$ не является дистанционно-транзитивным при $k>1$.

13. Граф $\bar{\Gamma}_{2 k-1 \cup 2 k \cup n-2 k+1 \cup n-2 k+2}$ при чётном $n$. Пусть $k=1$. Из работы [5] следует, что

$$
\rho_{1,2}^{(5)}(\alpha, \beta)=\min \{\lceil\|\alpha+\beta\| / 2\rceil, n / 2+1-\lceil\|\alpha+\beta\| / 2\rceil\}
$$

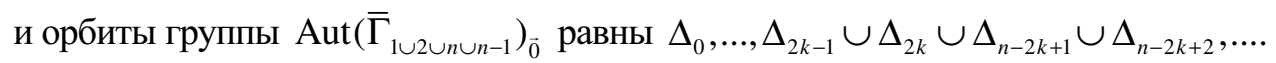

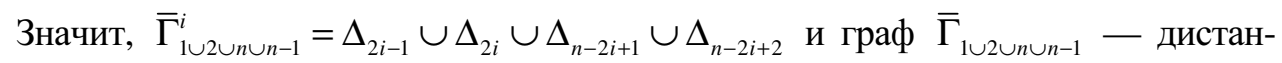
ционно-транзитивный.

При $k>1$ из работы [5] следует, что

$$
\Delta_{1} \cup \Delta_{2} \cup \Delta_{3} \cup \Delta_{4 k} \subseteq \bar{\Gamma}_{2 k-1 \cup 2 k \cup n-2 k+1 \cup n-2 k+2}^{2}(\overrightarrow{0}) \text { при } k \leq\lfloor n / 4\rfloor,
$$

а орбиты группы $\operatorname{Aut}\left(\bar{\Gamma}_{k \cup k+1 \cup n-k \cup n-k+1}\right)_{\overrightarrow{0}}$ равны

$$
\Delta_{0}, \Delta_{1} \cup \Delta_{2} \cup \Delta_{n-1} \cup \Delta_{n}, \ldots, \Delta_{2 i-1} \cup \Delta_{2 i} \cup \Delta_{n-2 i+1} \cup \Delta_{n-2 i+2}, \ldots .
$$

Таким образом, граф $\bar{\Gamma}_{2 k-1 \cup 2 k \cup n-2 k+1 \cup n-2 k+2}$ не является дистанционнотранзитивным при $k>1$.

14. Граф $\bar{\Gamma}_{2 k-1 \cup 2 k \cup n-2 k+1 \cup n-2 k}$ при нечётном $n$. Пусть $k=1$. Из работы [5] следует, что

$$
\left.\rho_{1,1}^{(5)} \alpha, \beta\right)=2 \text { при }\|\alpha+\beta\| \in\{3,4, n-4, n-3, n\},
$$

a орбиты группы $\operatorname{Aut}\left(\bar{\Gamma}_{1 \cup 2 \cup n-1 \cup n-2}\right)_{\overline{0}}$ равны $\Delta_{0}, \ldots, \Delta_{2 k-1} \cup \Delta_{2 k} \cup \Delta_{n-2 k} \cup \Delta_{n+1-2 k}, \ldots, \Delta_{n}$, то

$$
\bar{\Gamma}_{1 \cup 2 \cup n-2 \cup n-1}^{2}(\overrightarrow{0})=\left(\Delta_{3} \cup \Delta_{4} \cup \Delta_{n-3} \cup \Delta_{n-4}\right) \cup \Delta_{n} .
$$

Следовательно, граф $\bar{\Gamma}_{1 \cup 2 \cup n-1 \cup n-2}$ не является дистанционнотранзитивным.

При $k>1$ из работы [5] следует, что

$$
\Delta_{2} \cup \Delta_{3} \cup \Delta_{4 k} \subseteq \bar{\Gamma}_{2 k-1 \cup 2 k \cup n-2 k+1 \cup n-2 k}^{2}(\overrightarrow{0}) \text { при } k \leq\lfloor n / 4\rfloor,
$$

a орбиты группы $\operatorname{Aut}\left(\bar{\Gamma}_{2 k-1 \cup 2 k \cup n-2 k+1 \cup n-2 k}\right)_{\overline{0}}$ равны

$$
\Delta_{0}, \Delta_{1} \cup \Delta_{2} \cup \Delta_{n-1} \cup \Delta_{n-2}, \ldots, \Delta_{2 i-1} \cup \Delta_{2 i} \cup \Delta_{n-2 i} \cup \Delta_{n+1-2 i}, \ldots, \Delta_{n} .
$$


Таким образом, граф $\bar{\Gamma}_{2 k-1 \cup 2 k \cup n-2 k+1 \cup n-2 k}$ не является дистанционнотранзитивным.

Дистанционная транзитивность графов $\Gamma_{[0 \cup l \cup 2 \cup 3]}, \Gamma_{(0 \cup l \cup 2 \cup 3)}$ очевидна. Теорема доказана.

Отметим, что на рисунках 1 и 2 имеются пути от дистанционно-

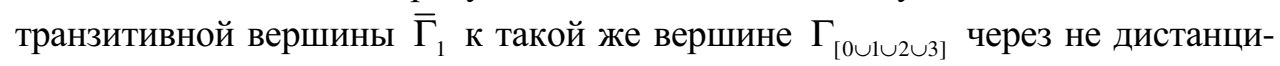
онно-транзитивные графы. При этом интранзитивность группы автоморфизмов наблюдается уже на путях длины два.

Следствие 2.3. При $n \geq 6$ следующие графы нечётных орбиталов не дистанционно-транзитивны:

1) $\bar{\Gamma}_{k}$ при нечётном $k \geq 3$;

2) $\bar{\Gamma}_{(1)}, \bar{\Gamma}_{(3)}$ при нечётном $n$;

3) $\bar{\Gamma}_{2 k-1 \cup 2 k}$ nрu $k \geq 2$;

4) $\bar{\Gamma}_{k \cup n-k}$ при нечётном $k \geq 1$;

5) $\bar{\Gamma}_{k \cup n-k+1}$ при нечётном $k \geq 2$;

6) $\bar{\Gamma}_{k \cup n-k-1}$ при нечётном $k \geq 1$;

7) $\bar{\Gamma}_{2 k-1 \cup 2 k \cup n-2 k+1 \cup n-2 k+2}$ при чётном $n$ и $k \geq 2$;

8) $\bar{\Gamma}_{2 k-1 \cup 2 k \cup n-2 k+1 \cup n-2 k}$ при нечётном $n u k \geq 1$;

9) $\bar{\Gamma}_{(1)}, \bar{\Gamma}_{(3)}$ при нечётном $n$.

Доказательство непосредственно следует из теоремы 2.2.

Чисто технически описываются изоморфные графы среди дистанционно-транзитивных графов нечётных орбиталов, приведенных в теореме 2.2 .

Утверждение 2.4. Пусть $n \geq 6$.

1. Графы $\bar{\Gamma}_{[1]}, \bar{\Gamma}_{[3]}$ изоморфны только при $n \equiv 0(\bmod 4)$.

2. Графы $\bar{\Gamma}_{(0 \cup 1)}, \bar{\Gamma}_{(2 \cup 3)}$ изоморфны только при $n \equiv 3(\bmod 4)$.

3. Графы $\bar{\Gamma}_{(1 \cup 2)}, \bar{\Gamma}_{(0 \cup 3)}$ изоморфны только при $n \equiv 1(\bmod 4)$.

4. Графы $\bar{\Gamma}_{[0 \cup 1]}, \bar{\Gamma}_{[2 \cup 3]}$ неизоморфны.

5. Графы $\bar{\Gamma}_{[1 \cup 2]}, \bar{\Gamma}_{[0 \cup 3]}$ неизоморфны.

У дистанционно-транзитивных графов степени всех вершин равны. Приведем степени вершин дистанционно-транзитивных графов нечётных орбиталов. 
Утверждение 2.5. При $n \geq 6$ параметры дистанционно-транзитивных графов нечётных орбиталов задаются следуюшими таблицами:

1) для любого $n$

\begin{tabular}{|c|c|c|}
\hline Граф & Степень вершины & Диаметр графа \\
\hline$\overline{\Gamma_{1}}$ & $n$ & $n$ \\
\hline $\bar{\Gamma}_{1 \cup 2}$ & $\left(\begin{array}{l}n \\
2\end{array}\right)+n$ & $\lceil n / 2\rceil$ \\
\hline $\bar{\Gamma}_{1 \cup n}$ & $n+1$ & $\lceil n / 2\rceil$ \\
\hline$\overline{\bar{\Gamma}_{[1]}}$ & $\sum_{k=0}^{\left\lfloor\frac{n-1}{4}\right\rfloor}\left(\begin{array}{c}n \\
4 k+1\end{array}\right)$ & 3 \\
\hline$\overline{\bar{\Gamma}_{[3]}}$ & $\sum_{k=0}^{\left\lfloor\frac{n-3}{4}\right\rfloor}\left(\begin{array}{c}n \\
4 k+3\end{array}\right)$ & 3 \\
\hline$\overline{\bar{\Gamma}_{[1 \cup 3]}}$ & $\sum_{k=0}^{\left\lfloor\frac{n-1}{4}\right\rfloor}\left(\begin{array}{c}n \\
4 k+1\end{array}\right)+\sum_{k=0}^{\left\lfloor\frac{n-3}{4}\right\rfloor}\left(\begin{array}{c}n \\
4 k+3\end{array}\right)$ & 2 \\
\hline $\bar{\Gamma}_{(0 \cup 1 \cup 2 \cup 3)}$ & $2^{n}-1$ & 2 \\
\hline $\bar{\Gamma}_{\text {[0ulU2U3] }}$ & $2^{n}$ & 1 \\
\hline
\end{tabular}

2) для нечётного $n$

\begin{tabular}{|c|c|c|}
\hline Граф & Степень вершины & Диаметр графа \\
\hline $\begin{array}{l}\bar{\Gamma}_{(1 \cup 2)} \\
\bar{\Gamma}_{(0 \cup 1)}\end{array}$ & $\sum_{k=0}^{\left\lfloor\frac{n-2}{4}\right\rfloor}\left(\begin{array}{c}n \\
4 k+1\end{array}\right)+\sum_{k=0}^{\left\lfloor\frac{n-3}{4}\right\rfloor}\left(\begin{array}{c}n \\
4 k+2\end{array}\right)$ & 3 \\
\hline $\bar{\Gamma}_{(2 \cup 3)}$ & $\sum_{k=0}^{\left\lfloor\frac{n-3}{4}\right\rfloor}\left(\begin{array}{c}n \\
4 k+2\end{array}\right)+\sum_{k=0}^{\left\lfloor\frac{n}{4}-1\right\rfloor}\left(\begin{array}{c}n \\
4 k+3\end{array}\right)$ & 3 \\
\hline $\bar{\Gamma}_{(0 \cup 3)}$ & $\sum_{k=1}^{\left\lfloor\frac{n-1}{4}\right\rfloor}\left(\begin{array}{c}n \\
4 k\end{array}\right)+\sum_{k=0}^{\left\lfloor\frac{n}{4}-1\right\rfloor}\left(\begin{array}{c}n \\
4 k+3\end{array}\right)$ & 3 \\
\hline $\bar{\Gamma}_{(1 \cup 3)}$ & {$\left[\sum_{k=0}^{\left\lfloor\frac{n-2}{4}\right\rfloor}\left(\begin{array}{c}n \\
4 k+1\end{array}\right)+\sum_{k=0}^{\left\lfloor\frac{n}{4}-1\right\rfloor}\left(\begin{array}{c}n \\
4 k+3\end{array}\right)\right.$} & 3 \\
\hline
\end{tabular}


3) для чётного $n$

\begin{tabular}{|c|c|c|}
\hline Граф & Степень вершины & Диаметр графа \\
\hline $\bar{\Gamma}_{[1 \cup 2]} \bar{\Gamma}_{[0 \cup 1]}$ & $\sum_{k=0}^{\left\lfloor\frac{n-1}{4}\right\rfloor}\left(\begin{array}{c}n \\
4 k+1\end{array}\right)+\sum_{k=0}^{\left\lfloor\frac{n-2}{4}\right\rfloor}\left(\begin{array}{c}n \\
4 k+2\end{array}\right)$ & 2 \\
\hline $\bar{\Gamma}_{[2 \cup 3]}$ & $\sum_{k=0}^{\left\lfloor\frac{n-2}{4}\right\rfloor}\left(\begin{array}{c}n \\
4 k+2\end{array}\right)+\sum_{k=0}^{\left\lfloor\frac{n-3}{4}\right\rfloor}\left(\begin{array}{c}n \\
4 k+3\end{array}\right)$ & 2 \\
\hline $\bar{\Gamma}_{[0 \cup 3]}$ & $\sum_{k=1}^{\left\lfloor\frac{n}{4}\right\rfloor}\left(\begin{array}{c}n \\
4 k\end{array}\right)+\sum_{k=0}^{\left\lfloor\frac{n-3}{4}\right\rfloor}\left(\begin{array}{c}n \\
4 k+3\end{array}\right)$ & 2 \\
\hline $\bar{\Gamma}_{1 \cup 2 \cup n \cup n-1}$ & $2 n+1+\left(\begin{array}{l}n \\
2\end{array}\right)$ & $\lceil n / 2\rceil$ \\
\hline
\end{tabular}

Доказательство следует из работы [5].

\section{3. Фактор-метрики дистанционно-транзитивных графов}

Пусть $\Gamma-$ связный граф диаметра $d \geq 1$ с множеством вершин $X$ и естественной метрикой $\rho$. На множестве $X$ зададим бинарное отношение $t_{r}$, полагая $v t_{r} \alpha$ тогда и только тогда, когда $\rho(\alpha, v)=r$. Для некоторых классов графов существует такое значение $r \in\{\overline{1, d}\}$, что отношение $l_{r}$ становится отношением эквивалентности. К таким классам графов относятся, например, антиподальные и двудольные графы. В случае, когда $t_{r}$ есть отношение эквивалентности, обозначим через $t_{r}$ число классов эквивалентности, а через $X^{(i)}$ - класс эквивалентности, $i \in\left\{\overline{1, t_{r}}\right\}$.

Приведем определение двудольных и антиподальных графов с помощью бинарного отношения $t_{r}$. Граф $\Gamma$ называется двудольным, если $l_{2}$ есть отношение эквивалентности. В частности, классы эквивалентности $l_{2}$ называются долями и $t_{2}=2$. Граф $\Gamma$ называется антиподальным, если $t_{d}$ есть отношение эквивалентности. В частности, классы эквивалентности $l_{d}$ называются классами антиподальности.

Пусть $\rho$ - естественная метрика графа $\Gamma$, и $t_{r}, r \in\{2, d\}$, является отношением эквивалентности. Определим степень графа $\Gamma^{(2)}$ связного графа Г следующим образом. Если $r=2$, то множеством вершин графа $\Gamma^{(2)}$ 
является класс эквивалентности $X^{(1)}$, а множеством ребер $\left\{(\alpha, \beta) \in X^{(1)} \times X^{(1)} \mid \rho(\alpha, \beta)=2\right\}$. Метрику графа $\Gamma^{(2)}$ обозначим через $\bar{\rho}_{\text {д }}$.

Если $r=d$, то множеством вершин графа $\bar{\Gamma}^{(2)}$ являются классы эквивалентности $X^{(1)}, \ldots, X^{\left(t_{r}\right)}$, а множеством ребер $\left\{\left(X^{(i)}, X^{(j)}\right) \mid \exists(\alpha, \beta) \in X^{(i)} \times X^{(j)}: \rho(\alpha, \beta)=1\right\}$. Известно [10], если $\Gamma-$ двудольный дистанционно-транзитивный граф, то графы

$$
\begin{gathered}
\Gamma^{(2)}=\left(X^{(1)},\left\{(\alpha, \beta) \in X^{(1)} \times X^{(1)} \mid \rho(\alpha, \beta)=2\right\}\right), \\
\Gamma^{\prime}=\left(X^{(2)},\left\{(\alpha, \beta) \in X^{(2)} \times X^{(2)} \mid \rho(\alpha, \beta)=2\right\}\right),
\end{gathered}
$$

порождаемые разными долями $X^{(1)}, X^{(2)}$, являются изоморфными дистанционно-транзитивными графами диаметра $\lfloor d / 2\rfloor$. Граф $\Gamma^{(2)}$ также дистанционнотранзитивен диаметра $\lfloor d / 2\rfloor$. Метрику графа $\Gamma^{(2)}$ обозначим как $\bar{\rho}_{\mathrm{a}}$.

Для дистанционно-транзитивных графов с импримитивной группой автоморфизмов известен следующий результат.

Теорема 3.1 [15]. Конечный дистаниионно-транзитивный граф Г валентности больше двух с импримитивной группой автоморфизмов $\operatorname{Aut}(\Gamma)$ или двудольный, или антиподальный, причем блоками импримитивности $\operatorname{Aut}(\Gamma)$ являются доли для двудольного графа или классы антиподальности для антиподального графа.

Таким образом, если валентность дистанционно-транзитивного графа больше двух и задана система импримитивности его группы автоморфизмов, то двудольность и антиподальность графа доказываются несложно.

Дистанционно-транзитивные графы нечётных орбиталов (теорема 2.2) имеют валентность, равную длине подорбиты, а потому большую двух. Опишем метрики этих графов, получающиеся при факторизации по блокам импримитивности групп автоморфизмов, выявим двудольные и антиподальные графы среди них. Блоки импримитивности групп автоморфизмов описаны в работах [4] и [5]. В частности, описываются примитивные дистанционно-транзитивные графы нечётных орбиталов (с примитивной группой автоморфизмов) и импримитивные графы (с импримитивной группой автоморфизмов).

В таблице в столбце «тип» ставится символ «а», если граф антиподальный, и «д» - граф двудольный, иначе ставится прочерк. Также в таблице прочерки означают несуществование соответствующего графа, а значит, и фактор-метрики. Если граф не является дистанционно-транзитивным, то ему соответствует пустая ячейка. 
Теорема 3.2. При $n \geq 6$ дистанционно-транзитивные графы нечётных орбиталов подсхем схемы Хемминга обладают следующими свойствами, приведенными в таблице:

\begin{tabular}{|c|c|c|c|c|c|c|}
\hline \multirow[b]{2}{*}{ Граф } & \multicolumn{3}{|c|}{ нечетное $n$} & \multicolumn{3}{|c|}{ чётное $n$} \\
\hline & $\begin{array}{c}\text { тип: } \\
\text { a, д }\end{array}$ & $\bar{\rho}_{\mathrm{a}}$ & $\bar{\rho}_{\text {д }}$ & $\begin{array}{c}\text { тип: } \\
\text { а, д }\end{array}$ & $\bar{\rho}_{\mathrm{a}}$ & $\overline{\bar{\rho}_{\text {д }}}$ \\
\hline $\bar{\Gamma}_{1 \cup 2}$ & $\mathrm{a}$ & $\rho_{1}^{(4)}$ & - & - & - & - \\
\hline $\bar{\Gamma}_{1 \cup n}$ & д & - & $\rho_{1,1}^{(5)}$ & - & - & - \\
\hline 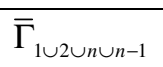 & & & & - & - & - \\
\hline $\bar{\Gamma}_{\text {[oul] }}$ & & & & - & - & - \\
\hline $\bar{\Gamma}_{[1 \cup 2]}$ & & & & - & - & - \\
\hline $\bar{\Gamma}_{(1 \cup 2)}$ & $\mathrm{a}$ & $\begin{array}{l}\rho_{2}^{(6)} \text { при } \\
n \equiv 1(\bmod 4) \\
\rho_{1,1}^{(2)} \text { при } \\
n \equiv 3(\bmod 4)\end{array}$ & - & & & \\
\hline $\bar{\Gamma}_{(0 \cup 1)}$ & $\mathrm{a}$ & $\begin{array}{l}\rho_{2}^{(6)} \text { при } \\
n \equiv 3(\bmod 4), \\
\rho_{0,1}^{(2)} \text { при } \\
n \equiv 1(\bmod 4)\end{array}$ & - & & & \\
\hline $\bar{\Gamma}_{(1 \cup 3)}$ & $\mathrm{a}$, д & $\rho_{2}^{(6)}$ & $\rho_{2}^{(6)}$ & & & \\
\hline $\bar{\Gamma}_{[1 \cup 3]}$ & д & - & $\rho_{2}^{(6)}$ & д & - & $\rho_{2}^{(6)}$ \\
\hline $\bar{\Gamma}_{[1]}$ & д & - & $\rho_{2}^{(6)}$ & д & - & $\overline{\rho_{2}^{(6)}}$ \\
\hline $\bar{\Gamma}_{[0 \cup 1 \cup 2 \cup 3]}$ & - & - & - & - & - & - \\
\hline $\bar{\Gamma}_{(0 \cup 1 \cup 2 \cup 3)}$ & $\mathrm{a}$ & $\rho_{2}^{(6)}$ & - & $\mathrm{a}$ & $\rho_{2}^{(6)}$ & - \\
\hline
\end{tabular}

Доказательство. Согласно теореме 2.2 достаточно описать блоки импримитивности групп автоморфизмов следующих графов:

a) $\bar{\Gamma}_{1}, \bar{\Gamma}_{1 \cup 2}, \bar{\Gamma}_{1 \cup n}, \bar{\Gamma}_{[1]}, \bar{\Gamma}_{[3]}, \bar{\Gamma}_{[1 \cup 3]}, \bar{\Gamma}_{[0 \cup 1 \cup 2 \cup 3]}, \bar{\Gamma}_{(0 \cup 1 \cup 2 \cup 3)}$ при любом $n \geq 6$;

б) $\bar{\Gamma}_{(0 \cup 1)}, \bar{\Gamma}_{(2 \cup 3)}, \bar{\Gamma}_{(1 \cup 2)}, \bar{\Gamma}_{(0 \cup 3)}, \bar{\Gamma}_{(1 \cup 3)}$ при нечётном $n \geq 6$;

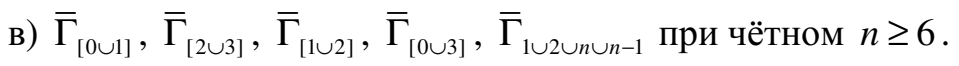


1. Граф $\bar{\Gamma}_{1 \cup 2}$. Если $n$ нечётно, то группа $\operatorname{Aut}\left(\bar{\Gamma}_{1 \cup 2}\right)$ импримитивна с системой импримитивности $\left\{(\alpha, \alpha+\overrightarrow{1}) \mid \alpha \in W_{n}\right\}$. Следовательно, граф $\bar{\Gamma}_{\text {l৩2 }}-$ антиподальный. Орбитами фактор-группы $\overline{\operatorname{Aut}\left(\bar{\Gamma}_{1 \cup 2}\right)_{\overline{0}}}$ являются множества $\Delta_{0}^{(n-1)}, \Delta_{1}^{(n-1)} \cup \Delta_{2}^{(n-1)}, \ldots, \Delta_{2 k-1}^{(n-1)} \cup \Delta_{2 k}^{(n-1)}, \ldots$. Значит, метрика графа $\bar{\Gamma}_{1 \cup 2}^{2}$ задаётся равенством $\bar{\rho}(\alpha, \beta)=\left\lceil\frac{\|\alpha+\beta\|}{2}\right\rceil, \alpha, \beta \in V_{n-1}$, и совпадает с метрикой $\rho_{1}^{(4)}$.

Если $n$ чётно, то группа $\operatorname{Aut}\left(\bar{\Gamma}_{1 \cup 2}\right)$ примитивна. Следовательно, граф $\bar{\Gamma}_{1 \cup 2}$ не является антиподальным или двудольным. Значит, у него нет фактор-метрик.

2. Граф $\bar{\Gamma}_{1 \cup n}$. Если $n$ нечётно, то группа $\operatorname{Aut}\left(\bar{\Gamma}_{1 \cup n}\right)$ импримитивна с системой импримитивности $\left\{W_{n}, W_{n}+\overrightarrow{1}\right\}$. Значит, граф $\bar{\Gamma}_{1 \cup n}-$ двудольный. Кроме того, граф $\bar{\Gamma}_{1 \cup n}^{(2)}$ связный с множеством вершин $W_{n}$ и метрикой $\bar{\rho}(\alpha, \beta)=\rho_{1,1}^{(3)}(\alpha, \beta) / 2, \alpha, \beta \in W_{n}$. Поскольку $\overline{\operatorname{Aut}\left(\bar{\Gamma}_{1 \cup n}\right)}=A S_{n-1}^{(2)}$, то факторметрика задаётся равенством

$$
\bar{\rho}(\alpha, \beta)=\min \{\lceil\|\alpha+\beta\| / 2\rceil,(n-1) / 2+1-\lceil\|\alpha+\beta\| / 2\rceil\}, \alpha, \beta \in V_{n-1},
$$

и совпадает с метрикой $\rho_{1,1}^{(5)}$.

Если $n$ чётно, то группа $\operatorname{Aut}\left(\bar{\Gamma}_{1 \cup n}\right)$ примитивна. Таким образом, граф $\bar{\Gamma}_{1 \cup n}$ не является антиподальным или двудольным.

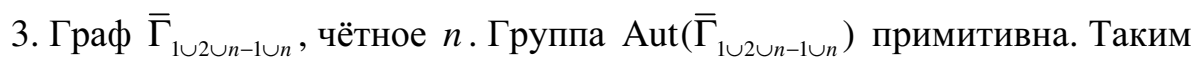
образом, граф $\bar{\Gamma}_{1 \cup 2 \cup n-1 \cup n}$ не является антиподальным или двудольным.

4. Графы $\bar{\Gamma}_{[0 \cup 1]}, \bar{\Gamma}_{[1 \cup 2]}$, чётное $n$. Поскольку группы $\operatorname{Aut}\left(\bar{\Gamma}_{[0 \cup 1]}\right)$, $\operatorname{Aut}\left(\bar{\Gamma}_{[1 \cup 2]}\right)$ примитивны, графы $\bar{\Gamma}_{[0 \cup 1]}, \bar{\Gamma}_{[1 \cup 2]}$ не являются антиподальными или двудольными.

5. Граф $\bar{\Gamma}_{(1 \cup 2)}$, нечётное $n$. Группа $\operatorname{Aut}\left(\bar{\Gamma}_{(1 \cup 2)}\right)$ импримитивна с системой импримитивности $\left\{(\alpha, \alpha+\overrightarrow{1}) \mid \alpha \in W_{n}\right\}$. Значит, граф $\bar{\Gamma}_{(1 \cup 2)}-$ антиподальный. Рассмотрим два случая.

5.1. Если $n \equiv 1(\bmod 4)$, то отображение $f_{1}: \alpha \rightarrow \alpha+\overrightarrow{1}$ обладает свойством $\left(\Lambda_{0} \cup \Lambda_{3}\right)^{f_{1}}=\left(\Lambda_{1} \cup \Lambda_{2}\right)$ и орбитами фактор-группы $\overline{\operatorname{Aut}\left(\bar{\Gamma}_{(1 \cup 2)}\right)_{\overrightarrow{0}}}$ явля- 
ются $\Delta_{0}^{(n-1)}, \Theta_{0}^{(n-1)} \cup \Theta_{1}^{(n-1)} \cup \Theta_{2}^{(n-1)} \cup \Theta_{3}^{(n-1)}$. Таким образом, метрика $\bar{\rho}$ графа $\bar{\Gamma}_{\text {(1ч2) }}^{2}$ тривиальная, т. е. $\bar{\rho}(\alpha, \beta)=1$ для любых $\alpha, \beta \in V_{n-1}, \alpha \neq \beta$.

5.2. Если $n \equiv 3(\bmod 4)$, то отображение $f_{1}: \alpha \rightarrow \alpha+\overrightarrow{1}$ обладает свойством $\Lambda_{0}^{f_{1}}=\Lambda_{3}, \Lambda_{1}^{f_{1}}=\Lambda_{2}$ и $\Delta_{0}^{(n-1)}, \Theta_{1}^{(n-1)} \cup \Theta_{2}^{(n-1)}, \Theta_{0}^{(n-1)} \cup \Theta_{3}^{(n-1)}$ 一 орбиты фактор-группы $\overline{\operatorname{Aut}\left(\bar{\Gamma}_{(1 \cup 2)}\right)}$. Значит, метрика графа $\bar{\Gamma}_{(1 \cup 2)}^{2}$ задаётся равенством

$$
\bar{\rho}(\alpha, \beta)= \begin{cases}1, & \|\alpha+\beta\| \equiv 1,2(\bmod 4), \\ 2, & \|\alpha+\beta\| \equiv 0,3(\bmod 4)\end{cases}
$$

для любых $\alpha, \beta \in V_{n-1}, \alpha \neq \beta$, и совпадает с метрикой $\rho_{1,1}^{(2)}$.

6. Граф $\bar{\Gamma}_{(0 \cup 1)}$, нечётное $n$. Группа $\operatorname{Aut}\left(\bar{\Gamma}_{(0 \cup 1)}\right)$ импримитивна с системой импримитивности $\left\{(\alpha, \alpha+\overrightarrow{1}) \mid \alpha \in W_{n}\right\}$. Значит, граф $\bar{\Gamma}_{(0 \cup 1)}-$ антиподальный.

6.1. Если $n \equiv 1(\bmod 4)$, то отображение $f_{1}: \alpha \rightarrow \alpha+\overrightarrow{1}$ обладает свойством $\Lambda_{0}^{f_{1}}=\Lambda_{1}, \Lambda_{2}^{f_{1}}=\Lambda_{3}$ и орбитами фактор-группы $\operatorname{Aut}\left(\bar{\Gamma}_{(0 \cup 1)}\right)_{\overline{0}}$ являются $\Delta_{0}^{(n-1)}, \quad \Theta_{0}^{(n-1)} \cup \Theta_{1}^{(n-1)}, \Theta_{2}^{(n-1)} \cup \Theta_{3}^{(n-1)}$. Таким образом, метрика графа $\bar{\Gamma}_{(0 \cup 1)}^{(2)}$ задаётся равенством

$$
\bar{\rho}(\alpha, \beta)= \begin{cases}1, & \|\alpha+\beta\| \equiv 0,1(\bmod 4), \\ 2, & \|\alpha+\beta\| \equiv 2,3(\bmod 4)\end{cases}
$$

для любых $\alpha, \beta \in V_{n-1}, \alpha \neq \beta$.

6.2. Если $n \equiv 3(\bmod 4)$, то отображение $f_{1}: \alpha \rightarrow \alpha+\overrightarrow{1}$ обладает свойством $\left(\Lambda_{0} \cup \Lambda_{1}\right)^{f_{1}}=\Lambda_{2} \cup \Lambda_{3}$ и орбитами фактор-группы $\operatorname{Aut}\left(\bar{\Gamma}_{(0 \cup 1)}\right)_{\overline{0}}$ являются $\Delta_{0}^{(n-1)}, \quad \Theta_{0}^{(n-1)} \cup \Theta_{1}^{(n-1)} \cup \Theta_{2}^{(n-1)} \cup \Theta_{3}^{(n-1)}$. Следовательно, метрика графа $\bar{\Gamma}_{(0 \cup 1)}$ тривиальная.

7. Граф $\bar{\Gamma}_{1 \cup 3}$, нечётное $n$. Группа $\operatorname{Aut}\left(\bar{\Gamma}_{1 \cup 3}\right)$ импримитивна с системами импримитивности $\left\{(\alpha, \alpha+\overrightarrow{1}) \mid \alpha \in W_{n}\right\}$ и $\left\{W_{n}, W_{n}+\overrightarrow{1}\right\}$. Следовательно, граф $\bar{\Gamma}_{1 \cup 3}-$ двудольный и антиподальный. 
7.1. Орбитами фактор-группы $\overline{\operatorname{Aut}\left(\bar{\Gamma}_{1 \cup 3}\right)_{0}}$, полученной факторизацией группы $\operatorname{Aut}\left(\bar{\Gamma}_{1 \cup 3}\right)$ по системе импримитивности $\left\{(\alpha, \alpha+\overrightarrow{1}) \mid \alpha \in W_{n}\right\}$, являются $\Delta_{0}^{(n-1)}, \Theta_{1}^{(n-1)} \cup \Theta_{3}^{(n-1)} \cup \Theta_{0}^{(n-1)} \cup \Theta_{2}^{(n-1)}$. Значит, метрика графа $\bar{\Gamma}_{1 \cup 3}$ тривиальна.

7.2. Фактор-группа $\overline{\operatorname{Aut}\left(\bar{\Gamma}_{1 \cup 3}\right)_{\overline{0}}}$, полученная факторизацией группы $\operatorname{Aut}\left(\bar{\Gamma}_{1 \cup 3}\right)$ по системе импримитивности $\left\{W_{n}, W_{n}+\overrightarrow{1}\right\}$, изоморфна $S_{2^{n-1}}$. Следовательно, фактор-метрика в этом случае также тривиальна.

8. Графы $\bar{\Gamma}_{[1 \cup 3]}, \bar{\Gamma}_{[1]}$. Для любого $n$ группы $\operatorname{Aut}\left(\bar{\Gamma}_{[1 \cup 3]}\right)$, $\operatorname{Aut}\left(\bar{\Gamma}_{[1]}\right)$ импримитивны с системой импримитивности $\left\{W_{n}, W_{n}^{\prime}\right\}$, где $W_{n}^{\prime}=V_{n} \backslash W_{n}$.

Следовательно, графы $\bar{\Gamma}_{[1 \cup 3]}, \bar{\Gamma}_{[1]}-$ двудольные. Нетрудно убедиться, что фактор-метрики графов $\bar{\Gamma}_{[1 \cup 3]}^{2}, \bar{\Gamma}_{[1]}^{2}$ тривиальны.

9. Граф $\bar{\Gamma}_{[0 \cup l \cup 2 \cup 3]}$. Для любого $n$ группа $\operatorname{Aut}\left(\bar{\Gamma}_{[0 \cup l \cup 2 \cup 3]}\right)$ примитивна.

10. Граф $\bar{\Gamma}_{(0 \cup 1 \cup 2 \cup 3)}$. Для любого $n$ группа $\operatorname{Aut}\left(\bar{\Gamma}_{(0 \cup l \cup 2 \cup 3)}\right)$ импримитивна с системой импримитивности $\left\{(\alpha, \alpha+\overrightarrow{1}) \mid \alpha \in V_{n}\right\}$. Значит, граф $\bar{\Gamma}_{(0 \cup l \cup 2 \cup 3)}-$ антиподальный с тривиальной метрикой. Утверждение доказано.

Таким образом, в теореме 3.2 установлена связь между подметриками и их фактор-метриками для дистанционно-транзитивных графов орбиталов при различных значениях $n$, а также описаны двудольные и антиподальные графы орбиталов.

Напомним [1], что если граф первого орбитала Г является двудольным, то порождаемая степенями его матрицы смежности схема отношений $H$ называется двудольной.

На множестве схем отношений определим унарную операцию «двудольности» - следующим образом:

a) если $H$ - двудольная схема, то $-H$ задается степенями матрицы смежности графа $\bar{\Gamma}^{2}$, порожденного отношением эквивалентности $l_{2}$;

б) если $H$ не является двудольной схемой, то $\bullet H=H$.

Для двудольной схемы $H$ схема $-H$ называется (см., например, [1]) половинной схемой двудольной схемы.

Если граф нечётного орбитала Г является антиподальным, то схема отношений $H$, порождаемая степенями его матрицы смежности, называется антиподальной [1]. 
На множестве схем отношений определим унарную операцию «аниподальности» $\odot$ следующим образом:

a) если $H$ - антиподальная схема, то схема $\odot H$ задается степенями матрицы смежности графа $\bar{\Gamma}=\Gamma^{[2]}$, порожденного отношением эквивалентности $l_{d}$, где $d-$ диаметр графа $\bar{\Gamma}$;

б) если $H$ не является антиподальной схемой, то $\odot H=H$.

Для антиподальной схемы $H$ схема $\odot H$ называется (см., например, [1]) производной схемой антиподальной схемы.

Теорема. 3.3. Множество подсхем схемы Хемминга замкнуто относительно унарных операций « $\boldsymbol{\bullet} » u$ « $\odot$ ».

Доказательство следует из утверждения 3.2 и работы [4].

Из теоремы 3.3 следует отсутствие новых классов подметрик среди полученных фактор-метрик дистанционно-транзитивных графов, отличных от рассмотренных в работе [5].

\section{4. Дистанционно-регулярные графы нечетных орбиталов}

Кроме дистанционно-транзитивных графов к важным классам графов относятся также дистанционно-регулярные графы. Любой дистанционнотранзитивный граф является дистанционно-регулярным, но обратное неверно.

Рассмотрим произвольный граф $\Gamma=(X, Y)$ диаметра $d$ с кратчайшим расстоянием $\rho$ между вершинами. Для произвольных вершин $u, v \in X$, $\rho(u, v)=j$, обозначим

$$
p_{i, h}^{(j)}(u, v)=|\{w \in X \mid \rho(u, w)=i, \rho(w, v)=h\}|, \quad i, j, h \in\{\overline{1, d}\} .
$$

Напомним, что граф $\Gamma=(X, Y)$ называется дистанционно-регулярным, если для любого $j \in\{\overline{1, d}\}$ и любых вершин $u, v, u^{\prime}, v^{\prime} \in X, \rho(u, v)=$ $=\rho\left(u^{\prime}, v^{\prime}\right)=j$, выполняются равенства $p_{1, h}^{(j)}(u, v)=p_{1, h}^{(j)}\left(u^{\prime}, v^{\prime}\right)=p_{h}^{(j)}$, где

$$
h \in\left\{\begin{array}{l}
\{j-1, j, j+1\}, \text { если } 2 \leq j \leq d-1, \\
\{1,2\}, \text { если } j=1, \\
\{d-1, d\}, \text { если } j=d,
\end{array}\right.
$$

т. е. значение величины $p_{1, h}^{(j)}$ не зависит от конкретных вершин, находящихся на расстоянии $j$ в графе $\Gamma$.

Приведем дистанционно-регулярные графы нечётных орбиталов подсхем схемы Хемминга. 
Теорема 4.1. Среди нечётных орбиталов подсхем схемы Хемминга дистанционно-регулярными графами являются только дистанционнотранзитивные графы.

Доказательство. Достаточно рассмотреть только графы, приведенные в следствии 2.3 и не являющиеся дистанционно-транзитивными.

Пусть $\mu-$ подметрика некоторой схемы Хемминга, $\|\alpha\|_{\mu}=\mu(\alpha, \overrightarrow{0})$. Поскольку

$$
\mu(\alpha, \beta)=\mu(\overrightarrow{0}, \alpha+\beta)=\|\alpha+\beta\|_{\mu}
$$

для произвольных вершин $\alpha, \beta \in V_{n}$, то для доказательства отсутствия дистанционной регулярности достаточно показать зависимость величины

$$
p_{1, h}^{(j)}(\overrightarrow{0}, \theta)=\left\{\left\{\gamma \in V_{n} \mid\|\gamma\|_{\mu}=1,\|\theta+\gamma\|_{\mu}=h\right\} \mid\right.
$$

от конкретного $\theta \in V_{n},\|\theta\|_{\mu}=j$. При доказательстве дистанционной нерегулярности для каждого из графов будем находить величины $p_{1,1}^{(2)}\left(\overrightarrow{0}, \theta_{1}\right), p_{1,1}^{(2)}\left(\overrightarrow{0}, \theta_{2}\right)$ для двух разных векторов $\theta_{1}, \theta_{2} \in V_{n},\left\|\theta_{1}\right\|_{\mu}=\left\|\theta_{2}\right\|_{\mu}=2$.

Пусть $\varepsilon_{t}=(0 . .010 \ldots 0)$ - векторы-строки из пространства $V_{n}, t \in\{\overline{1, n}\}$, $\lambda_{t}=\sum_{i=1}^{t} \varepsilon_{i}, t \geq 1$.

Будем придерживаться таких же обозначений, как в доказательстве теоремы 2.2.

1. Граф $\bar{\Gamma}_{k}, k \geq 3, \mu=\chi_{k}$. Справедливы соотношения:

$$
\begin{gathered}
\bar{\Gamma}_{k}(\overrightarrow{0})=\Delta_{k}, \\
\Delta_{2} \cup \Delta_{2 k} \subseteq \bar{\Gamma}_{k}^{2}(\overrightarrow{0}) \text { при } k \leq\lfloor n / 2\rfloor, \\
\Delta_{2} \cup \Delta_{2(n-k)} \subseteq \bar{\Gamma}_{k}^{2}(\overrightarrow{0}) \text { при } k>\lfloor n / 2\rfloor .
\end{gathered}
$$

Пусть $\theta_{1}=\lambda_{2}$,

$$
\theta_{2}=\left\{\begin{array}{l}
\lambda_{2 k}, \text { если } k \leq\lfloor n / 2\rfloor, \\
\lambda_{2(n-k)}, \text { если } k>\lfloor n / 2\rfloor .
\end{array}\right.
$$

Нетрудно убедиться в том, что для любого вектора $\theta \in \Delta_{j}$ выполняется равенство

$$
\left|\left\{\gamma \in V_{n} \mid\|\gamma\|=h,\|\theta+\gamma\|=l\right\}\right|=\left(\begin{array}{c}
j \\
\frac{h+j-l}{2}
\end{array}\right)\left(\begin{array}{c}
n-j \\
\frac{h+l-j}{2}
\end{array}\right) .
$$


Таким образом,

$$
\begin{gathered}
p_{1,1}^{(2)}\left(\overrightarrow{0}, \theta_{1}\right)=\left(\begin{array}{l}
2 \\
1
\end{array}\right)\left(\begin{array}{c}
n-2 \\
k-1
\end{array}\right), \\
p_{1,1}^{(2)}\left(\overrightarrow{0}, \theta_{2}\right)=\left\{\begin{array}{l}
\left(\begin{array}{c}
2 k \\
k
\end{array}\right), \text { если } k \leq\lfloor n / 2\rfloor, \\
\left(\begin{array}{c}
2(n-k) \\
n-k
\end{array}\right)\left(\begin{array}{c}
n-2(n-k) \\
k-(n-k)
\end{array}\right), \text { если } k>\lfloor n / 2\rfloor .
\end{array}\right.
\end{gathered}
$$

Поскольку $p_{1,1}^{(2)}\left(\overrightarrow{0}, \theta_{1}\right) \neq p_{1,1}^{(2)}\left(\overrightarrow{0}, \theta_{2}\right)$, граф $\bar{\Gamma}_{k}$ не является дистанционнорегулярным.

2. Графы $\bar{\Gamma}_{(1)}, \bar{\Gamma}_{(3)}$ при нечётном $n$. Для $k \in\{1,3\}$ справедливы соотношения:

$$
\bar{\Gamma}_{k}(\overrightarrow{0})=\Lambda_{k}, \bar{\Gamma}_{(k)}^{2}(\overrightarrow{0})=\Lambda_{2} \cup \Lambda_{0}
$$

Положим $\theta_{1}=\lambda_{2}, \theta_{2}=\lambda_{4}$. Нетрудно убедиться в справедливости равенств

$$
\begin{gathered}
p_{1,1}^{(2)}\left(\overrightarrow{0}, \theta_{1}\right)=\sum_{i=0}^{\lfloor(n-1-k) / 4\rfloor}\left(\begin{array}{l}
2 \\
1
\end{array}\right)\left(\begin{array}{c}
n-2 \\
4 i+k-1
\end{array}\right), \\
p_{1,1}^{(2)}\left(\overrightarrow{0}, \theta_{2}\right)=2 \sum_{i=0}^{\lfloor(n-4-k) / 4\rfloor}\left(\begin{array}{c}
n-4 \\
4 i+k
\end{array}\right)+\sum_{i=0}^{\lfloor(n-2-k) / 4\rfloor}\left(\begin{array}{l}
4 \\
2
\end{array}\right)\left(\begin{array}{c}
n-4 \\
4 i+k-2
\end{array}\right)
\end{gathered}
$$

и в том, что $p_{1,1}^{(2)}\left(\overrightarrow{0}, \theta_{1}\right) \neq p_{1,1}^{(2)}\left(\overrightarrow{0}, \theta_{2}\right)$. Следовательно, графы $\bar{\Gamma}_{(1)}, \bar{\Gamma}_{(3)}$ не являются дистанционно-регулярными.

3. Граф $\bar{\Gamma}_{k \cup n-k}$. Справедливы соотношения:

$$
\begin{gathered}
\bar{\Gamma}_{1 \cup n-1}^{2}(\overrightarrow{0})=\left(\Delta_{2} \cup \Delta_{n-2}\right) \cup \Delta_{n} ; \\
\Delta_{2} \cup \Delta_{n} \subseteq \bar{\Gamma}_{k \cup n-k}^{2}(\overrightarrow{0}) .
\end{gathered}
$$

Положим $\theta_{1}=\lambda_{2}, \theta_{2}=\lambda_{n}$. Применяя равенство (4.1), получаем

$$
p_{1,1}^{(2)}\left(\overrightarrow{0}, \theta_{2}\right)=\left(\begin{array}{l}
n \\
k
\end{array}\right)+\left(\begin{array}{c}
n \\
n-k
\end{array}\right)=2\left(\begin{array}{l}
n \\
k
\end{array}\right),
$$




$$
p_{1,1}^{(2)}\left(\overrightarrow{0}, \theta_{1}\right)=\left\{\begin{array}{l}
\left(\begin{array}{l}
2 \\
1
\end{array}\right)\left(\begin{array}{l}
n-2 \\
k-1
\end{array}\right)+\left(\begin{array}{l}
2 \\
1
\end{array}\right)\left(\begin{array}{c}
n-2 \\
n-(k-1)
\end{array}\right), \text { если } 2 k \neq n-2, \\
\left(\begin{array}{l}
2 \\
1
\end{array}\right)\left(\begin{array}{l}
n-2 \\
k-1
\end{array}\right)+\left(\begin{array}{l}
2 \\
1
\end{array}\right)\left(\begin{array}{c}
n-2 \\
n-(k-1)
\end{array}\right)+\left(\begin{array}{c}
n-2 \\
k
\end{array}\right)+\left(\begin{array}{c}
n-2 \\
n-k-2
\end{array}\right), \text { если } 2 k=n-2,
\end{array}\right.
$$

T. e.

$$
p_{1,1}^{(2)}\left(\overrightarrow{0}, \theta_{1}\right)=\left\{\begin{array}{l}
2\left(\begin{array}{c}
n-2 \\
k-1
\end{array}\right)+2\left(\begin{array}{c}
n-2 \\
k-3
\end{array}\right), \text { если } 2 k \neq n-2, \\
2\left(\begin{array}{c}
n-1 \\
k
\end{array}\right)+2\left(\begin{array}{c}
n-2 \\
k-3
\end{array}\right), \text { если } 2 k=n-2,
\end{array}\right.
$$

Значит, $p_{1,1}^{(2)}\left(\overrightarrow{0}, \theta_{1}\right) \neq p_{1,1}^{(2)}\left(\overrightarrow{0}, \theta_{2}\right)$ и граф $\bar{\Gamma}_{k \cup n-k}$ не является дистанционнорегулярным.

4. Граф $\bar{\Gamma}_{k \cup n-k+1}, k>1$. Очевидно, что достаточно рассмотреть только $k \leq\lfloor n / 2\rfloor$. Справедливо соотношение:

$$
\Delta_{2} \cup \Delta_{4} \cup \Delta_{2 k} \subseteq \bar{\Gamma}_{k \cup n-k+1}^{2}(\overrightarrow{0}) \text { при } k \leq\lfloor n / 2\rfloor .
$$

Положим $\theta_{1}=\lambda_{2}, \theta_{2}=\lambda_{4}$. Применяя равенство (4.1) и используя свойства биномиальных коэффициентов, получаем:

$$
\begin{aligned}
& p_{1,1}^{(2)}\left(\overrightarrow{0}, \theta_{1}\right)=\left\{\begin{array}{l}
2\left(\begin{array}{l}
n-1 \\
k-1
\end{array}\right), \text { если } 2 k \neq n-1, \\
\left(\begin{array}{l}
n \\
k
\end{array}\right)+\left(\begin{array}{l}
n-1 \\
k-1
\end{array}\right), \text { если } 2 k=n-1,
\end{array}\right. \\
& \int\left(\begin{array}{l}
4 \\
2
\end{array}\right)\left(\begin{array}{l}
n-3 \\
k-2
\end{array}\right)+\left(\begin{array}{c}
n-3 \\
k
\end{array}\right), \text { если } 2 k=n-3, \\
& p_{1,1}^{(2)}\left(\overrightarrow{0}, \theta_{2}\right)=\left\{\left(\begin{array}{l}
4 \\
2
\end{array}\right)\left(\begin{array}{l}
n-3 \\
k-2
\end{array}\right)+4\left(\begin{array}{l}
n-3 \\
k-1
\end{array}\right) \text {, если } 2 k=n-3,\right. \\
& \left(\begin{array}{l}
4 \\
2
\end{array}\right)\left(\begin{array}{l}
n-3 \\
k-2
\end{array}\right) \text { иначе. }
\end{aligned}
$$


Нетрудно убедиться в том, что $p_{1,1}^{(2)}\left(\overrightarrow{0}, \theta_{1}\right) \neq p_{1,1}^{(2)}\left(\overrightarrow{0}, \theta_{2}\right)$. Значит, граф $\bar{\Gamma}_{k \cup n-k+1}$ при $k>1$ не является дистанционно-регулярным.

5. Граф $\bar{\Gamma}_{k \cup n-k-1}$. Очевидно, что достаточно рассмотреть только $k \leq\lfloor n / 2\rfloor, k$ или $n-k-1$ нечётные. Справедливо соотношение:

$$
\Delta_{2} \cup \Delta_{4} \cup \Delta_{2 k} \subseteq \bar{\Gamma}_{k \cup n-k-1}^{2}(\overrightarrow{0}) \text { при } k \leq\lfloor n / 2\rfloor .
$$

Положим $\theta_{1}=\lambda_{2}, \theta_{2}=\lambda_{4}$. Применяя равенство (4.1) и используя свойства биномиальных коэффициентов, получаем:

$$
\begin{aligned}
& p_{1,1}^{(2)}\left(\overrightarrow{0}, \theta_{1}\right)=\left\{\begin{array}{l}
2\left(\begin{array}{c}
n-1 \\
k
\end{array}\right), \text { если } 2 k \neq n-3, \\
\left(\begin{array}{c}
n-1 \\
k
\end{array}\right)+\left(\begin{array}{c}
n \\
k+1
\end{array}\right), \text { если } 2 k=n-3,
\end{array}\right.
\end{aligned}
$$

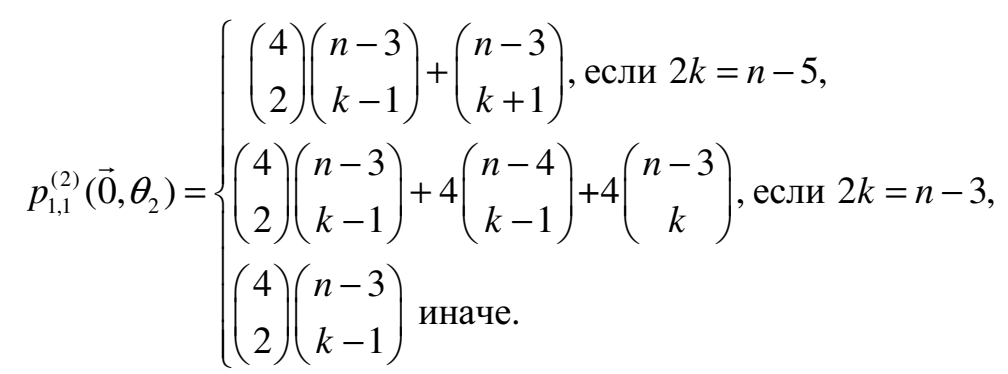

Нетрудно убедиться в том, что $p_{1,1}^{(2)}\left(\overrightarrow{0}, \theta_{1}\right) \neq p_{1,1}^{(2)}\left(\overrightarrow{0}, \theta_{2}\right)$. Значит, граф $\bar{\Gamma}_{k \cup n-k-1}$ не является дистанционно-регулярным.

6. $\bar{\Gamma}_{2 k-1 \cup 2 k}, k>1$. Справедливо соотношение

$$
\Delta_{2} \cup \Delta_{4} \subseteq \bar{\Gamma}_{2 k-1 \cup 2 k}^{2}(\overrightarrow{0}) .
$$

Положим $\theta_{1}=\lambda_{2}, \theta_{2}=\lambda_{4}$. Применяя равенство (4.1) и используя свойства биномиальных коэффициентов, получаем:

$$
\begin{aligned}
& p_{1,1}^{(2)}\left(\overrightarrow{0}, \theta_{1}\right)=\left(\begin{array}{l}
2 \\
1
\end{array}\right)\left(\begin{array}{c}
n-2 \\
2 k-2
\end{array}\right)+\left(\begin{array}{l}
2 \\
1
\end{array}\right)\left(\begin{array}{c}
n-2 \\
2 k-1
\end{array}\right)=2\left(\begin{array}{c}
n-1 \\
2 k-1
\end{array}\right), \\
& p_{1,1}^{(2)}\left(\overrightarrow{0}, \theta_{2}\right)=\left(\begin{array}{l}
4 \\
2
\end{array}\right)\left(\begin{array}{c}
n-4 \\
2 k-3
\end{array}\right)+\left(\begin{array}{l}
4 \\
2
\end{array}\right)\left(\begin{array}{c}
n-4 \\
2 k-2
\end{array}\right)=2\left(\begin{array}{c}
n-3 \\
2 k-2
\end{array}\right) .
\end{aligned}
$$


Нетрудно убедиться в том, что $p_{1,1}^{(2)}\left(\overrightarrow{0}, \theta_{1}\right) \neq p_{1,1}^{(2)}\left(\overrightarrow{0}, \theta_{2}\right)$. Значит, граф $\bar{\Gamma}_{2 k-1 \cup 2 k}$ при $k>1$ не является дистанционно-регулярным.

7. Граф $\bar{\Gamma}_{2 k-1 \cup 2 k \cup n-2 k+1 \cup n-2 k+2}$ при чётном $n, k>1$. Справедливо соотношение:

$$
\Delta_{2} \cup \Delta_{4} \subseteq \bar{\Gamma}_{2 k-1 \cup 2 k \cup n-2 k+1 \cup n-2 k+2}^{2}(\overrightarrow{0}) .
$$

Рассмотрим $\theta_{1}=\lambda_{2}, \theta_{2}=\lambda_{4}$. Применяя равенство (4.1) и используя свойства биномиальных коэффициентов, получаем:

$$
\begin{aligned}
& p_{1,1}^{(2)}\left(\overrightarrow{0}, \theta_{1}\right)=\left\{\begin{array}{l}
2\left(\begin{array}{c}
n \\
2 k-1
\end{array}\right), \text { если } n \neq 4 k, \\
\left(\begin{array}{c}
n \\
2 k-1
\end{array}\right)+\left(\begin{array}{c}
n+1 \\
2 k
\end{array}\right), \text { если } n=4 k,
\end{array}\right.
\end{aligned}
$$

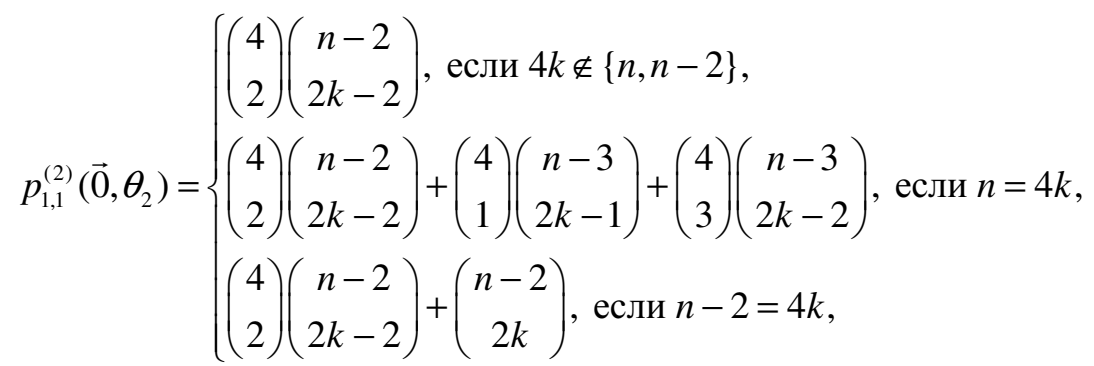

Нетрудно убедиться в том, что $p_{1,1}^{(2)}\left(\overrightarrow{0}, \theta_{1}\right) \neq p_{1,1}^{(2)}\left(\overrightarrow{0}, \theta_{2}\right)$. Значит, граф $\bar{\Gamma}_{2 k-1 \cup 2 k \cup n-2 k+1 \cup n-2 k+2}$ при чётном $n, k>1$, не является дистанционнорегулярным.

8. Граф $\bar{\Gamma}_{2 k-1 \cup 2 k \cup n-2 k+1 \cup n-2 k}$ при нечётном $n$. Справедливо соотношение:

$$
\Delta_{2} \cup \Delta_{4} \subseteq \bar{\Gamma}_{2 k-1 \cup 2 k \cup n-2 k+1 \cup n-2 k}^{2}(\overrightarrow{0}) \text {. }
$$

Положим $\theta_{1}=\lambda_{2}, \theta_{2}=\lambda_{4}$. Применяя равенство (4.1) и используя свойства биномиальных коэффициентов, получаем:

$$
p_{1,1}^{(2)}\left(\overrightarrow{0}, \theta_{1}\right)=\left\{\begin{array}{l}
4\left(\begin{array}{c}
n-1 \\
2 k-1
\end{array}\right), \text { если } n-1 \neq 4 k, \\
4\left(\begin{array}{c}
n-1 \\
2 k-1
\end{array}\right)+2\left(\begin{array}{c}
n-1 \\
2 k
\end{array}\right), \text { если } n-1=4 k,
\end{array}\right.
$$




$$
p_{1,1}^{(2)}\left(\overrightarrow{0}, \theta_{2}\right)=\left\{\begin{array}{l}
2\left(\begin{array}{l}
4 \\
2
\end{array}\right)\left(\begin{array}{c}
n-3 \\
2 k-2
\end{array}\right), \text { если } 4 k \notin\{n, n-2\}, \\
2\left(\begin{array}{l}
4 \\
2
\end{array}\right)\left(\begin{array}{c}
n-3 \\
2 k-2
\end{array}\right)+\left(\begin{array}{l}
4 \\
1
\end{array}\right)\left(\begin{array}{c}
n-3 \\
2 k-1
\end{array}\right)+\left(\begin{array}{l}
4 \\
3
\end{array}\right)\left(\begin{array}{c}
n-3 \\
2 k-1
\end{array}\right), \text { если } n-1=4 k, \\
2\left(\begin{array}{l}
4 \\
2
\end{array}\right)\left(\begin{array}{c}
n-3 \\
2 k-2
\end{array}\right)+2\left(\begin{array}{c}
n-3 \\
2 k
\end{array}\right), \text { если } n-3=4 k .
\end{array}\right.
$$

Нетрудно убедиться в том, что $p_{1,1}^{(2)}\left(\overrightarrow{0}, \theta_{1}\right) \neq p_{1,1}^{(2)}\left(\overrightarrow{0}, \theta_{2}\right)$. Значит, граф $\bar{\Gamma}_{2 k-1 \cup 2 k \cup n-2 k+1 \cup n-2 k}$ при нечётном $n$ не является дистанционно-регулярным. Теорема доказана.

\section{Список литературы}

1. Баннаи Э., Ито Т. Алгебраическая комбинаторика. - М.: Мир, 1987, $373 \mathrm{c.}$

2. Музычук M. E. Подсхемы схемы Хемминга. Исследования по алгебраической теории комбинаторных объектов. - В сб.: ВНИИ системных исследований. Труды семинара. - М., 1985, с. 49-76.

3. Погорелов Б. А. Подметрики метрики Хемминга и теорема А. А. Маркова. В сб.: Труды по дискретной математике. Т. 9. - М.: ФИЗМАТЛИТ, 2006.

4. Погорелов Б. А., Пудовкина М. А. Подметрики метрики Хемминга и преобразования, распространяющие искажения в заданное число раз. В сб.: Труды по дискретной математике. Т. 10. - М.: ФИЗМАТЛИТ, 2007.

5. Погорелов Б. А., Пудовкина М. А. Подметрики Хемминга и их группы изометрий. В сб.: Труды по дискретной математике. Т. 11. - М.: ФИЗМАТЛИТ, 2008.

6. Харари Ф. Теория графов. - М.: Мир, 1973, 300 с.

7. Brouwer A. E., Cohen A. M., Neumaier A. Distance-regular graphs. - Berlin: Springer-Verlag, 1989.

8. van Bon J. T. M., Brouwer A. E. The distance-regular antipodal covers of classical distance-regular graphs. - In: Colloq. Math. Soc. János Bolyai, Proc. Eger, 1987, p. 141-166.

9. Brouwer A. E., Koolen J. H. The distance-regular graphs of valency four. In: J. Algebr. Combin. 1999, v. 10, p. 5-24.

10. Cameron P. J. Permutation groups. - London Math. Soc. Student Texts. V. 45. In: Cambridge Univ. Press, 1999.

11. Godsil C. Association schemes. - Lect. notes, Univ. Waterloo, 2004. 
12. Ivanov A. A. Distance-transitive graphs and their classification. - In: Investigations in algebraic theory of combinatorial objects. - Dordrecht: Kluwer, 1994, p. 283-378.

13. Ivanov A. A. Liebler R., Penttila T., Praeger C. Antipodal distance-transitive covers of complete bipartite graphs. - European J. Combin., 1997, v. 18, p. 11-33.

14. Praeger C. E. Finite transitive permutation groups and bipartite vertextransitive graphs. - Illinois J. Math., 2003, v. 47, p. 461-475.

15. Smith D. H. Primitive and imprimitive graphs. - Quart. J. Math. Oxford, 1971, v. 22, p. 551-557.

16. Soicher $L$. H. The GRAPE package for GAP. - http://www.maths.qmul.ac.uk/ leonard/grape.

17. Soicher L. H. Three new distance-regular graphs. - Europ. J. Comb. 1993, v. 14, p. 501-505. 

